\title{
Providing public transport priority in the perimeter of urban networks: a bimodal strategy
}

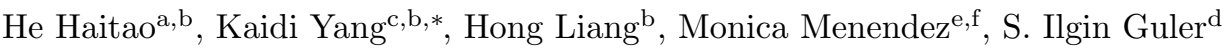 \\ ${ }^{a}$ School of Architecture, Building and Civil Engineering, Loughborough University, UK \\ ${ }^{b}$ Institute for Transport Planning and Systems, ETH Zurich, Switzerland \\ ${ }^{c}$ Department of Aeronautics and Astronautics, Stanford University, USA \\ ${ }^{d}$ Department of Civil and Environmental Engineering, The Pennsylvania State University, USA \\ ${ }^{e}$ Division of Engineering, New York University Abu Dhabi, United Arab Emirates \\ ${ }^{f}$ Tandon School of Engineering, New York University, USA
}

\begin{abstract}
Bimodal urban networks are complex systems operating within multiple constraints. This paper develops an integrated and systematic framework for the optimization of bimodal urban networks using 3D-MFDs, considering the complexities of bimodality. With the proposed framework, effective strategies can be designed for the planning, management, and control of bimodal networks. In particular, strategies to provide public transport priority on the network level can be holistically evaluated. We apply this methodological framework to propose, model, and analyze one such strategy to provide public transport priority in the perimeter of urban networks. The proposed strategy addresses a pressing problem of the existing perimeter control (i.e. gating) schemes: public transport vehicles will be queuing with the cars in the perimeter and hence blocked from entering the network. This impairs the service quality of public transport. Adopting our proposed strategy, the inflows of public transport and cars can be regulated independently (i.e. both inflows are controllable), the network traffic can be managed more efficiently, and public transport priority can be provided. The performance of the proposed strategy is evaluated both analytically and with simulations. Results show that the proposed strategy always performs better than existing perimeter control schemes in terms of passenger mobility. Most importantly, it differentiates the public transport mode and the car mode, with much smaller queueing time outside the network for public transport. This can shift the transportation system to a more sustainable state in the long run. Policy recommendations are provided for a large range of traffic scenarios.
\end{abstract}

Keywords: Public transport priority; Multimodal; Urban network; Optimization; 3D-MFD; Pre-signal

\section{Introduction}

Traffic congestion is an acute and persisting problem in many urban areas. Such congestion causes not only excessive delays, but also many externalities such as environmental impacts and reduced safety. The

\footnotetext{
* Corresponding author

Phone: +1 650-885-1186

Email address: kaidi.yang@stanford.edu (Kaidi Yang)
} 
existence of an invariant and reproducible Macroscopic Fundamental Diagram (MFD) proves there is a well-defined critical network vehicle density (or accumulation) (Mahmassani et al., 1987, Geroliminis and Daganzo, 2008; Daganzo and Geroliminis, 2008). At this critical density, the average flow of the network, which is proportional to the trip completion rate in the network, is optimum (Daganzo, 2007, Mahmassani et al. 2013). If this critical density is exceeded, the network enters the congested regime, where the average flow is reduced and vehicles suffer delay. In the worst case, such congestion could eventually lead to a gridlock. Therefore, it is beneficial to control the network density near the critical value, and it is crucial to ensure that this value is not substantially exceeded.

Various traffic management and control strategies have been implemented to achieve this goal. These strategies aim to maintain the network density near the critical value, especially during peak periods when the network is under excessive demand. One example of such strategies is perimeter congestion charging(Lindsey and Verhoef, 2001, Daganzo and Lehe, 2015, Yang et al., 2019b), implemented for example in London (Santos and Bhakar 2006). Another example is perimeter control (which is sometimes called 'gating'), implemented for example in Zurich (Ambühl et al., 2018b). This strategy regulates the transfer flows across the border based on real time feedback on traffic states. Recently, there have been intensive research efforts devoted to developing various perimeter control strategies, for single region (Keyvan-Ekbatani et al., 2012, 2015), for multi-region areas (Aboudolas and Geroliminis, 2013, Ramezani et al., 2015, Haddad and Zheng, 2018, Mohajerpoor et al., 2019 | Mariotte and Leclercq, 2019), or even as an alternative to dedicated bus lanes (Chiabaut et al. | 2018). However, while congestion pricing commonly differentiates between modes of transport by charging different prices (Santos and Bhakar, 2006, Wichiensin et al., 2007; Kottenhoff and Freij, 2009: Zheng et al., 2016, Pandey and Boyles, 2018), none of the perimeter control strategies developed so far have preferential treatment for different modes. In particular, they are not able to regulate the inflows of public transport vehicles and cars independently, but only the total vehicle flow, i.e. the two inflows are dependent. This has two major drawbacks.

First, when the two modes cannot be regulated independently, it is implicitly assumed that public transport (e.g. buses) is distributed among cars. Therefore, during peak periods, when cars are queued outside of a regulated perimeter, buses will also be restricted from entering the network, as they are queued among the cars. Evidently, this is not ideal in practice because bus priority cannot be provided, and buses will not be able to run on schedule. Compared to cars, public transport carries more passengers using less space. Therefore, in urban areas with limited space and high travel demand, public transport is more efficient. According to Smeed (1961, 1968); Loder et al. (2017), the greater the travel demand, the greater the share of public transport users must be to maintain a certain average speed. In the long run, promoting public transport in urban transportation networks can reduce passenger travel time (Hensher, 2001), relieve congestion (Adler and van Ommeren, 2016), boost economy (Chatman and Noland, 2011), and eventually lead to a more sustainable transportation system. Therefore, dedicated lanes are often implemented to provide public transport priority, so that public transport vehicles can jump the car queues without getting stuck in congestion. When such priority is provided at the regulated perimeter, it encourages more people to commute to the city center with public transport (Gonzales and Daganzo, 2012). Indeed, Geroliminis et al. (2014) suggests that such preferential treatment of public transport in network control strategies should be a research priority. Unfortunately, so far no existing perimeter control scheme is capable of capturing this.

Second, independent regulation of the two modes can be more efficient in reaching the target accumulations and achieving the traffic management and control goals. Unfortunately, the complex impact of multimodality on the network level traffic performance has not received much attention (Geroliminis et al. 2014 Loder et al. 2017), especially when deriving strategies for perimeter control. Only a few studies looked into perimeter control strategies for a bimodal network (Ampountolas et al. 2017) using multimodal MFDs. However, although the network in these models is bimodal, only the total boundary transfer flow is regulated in these strategies. These are referred to as single-mode strategies in this paper. In these strategies, public transport is assumed to be homogeneously distributed in the traffic stream in each regulated area (although this composition can vary with time and regions). Therefore, the composition of the inflow is determined by the 
composition of the incoming demand or queued vehicles in the perimeter, which cannot be directly controlled. This inflow composition might not be ideal for the traffic management and control goals. Perimeter control strategies would be more efficient when we can control the composition of the inflow, in addition to its magnitude. This paper proposes such perimeter control strategies where the inflows of the two modes can be regulated independently. These are referred to as bimodal strategies in this paper.

To realize a bimodal strategy, appropriate monitoring tools for bimodal networks are essential. Some recent research has focused on the development of such monitoring tools for bimodal networks. Chiabaut (2015) extends the concept of the MFD to account for the number of passengers by using the passenger macroscopic fundamental diagram (p-MFD). Geroliminis et al. (2014) proposes to use the 3D-MFD and the 3D-pMFD to represent the characteristics of bimodal networks. Some recent literature has already proven its existence with empirical evidence. For example, Loder et al. (2017) and Ambühl et al. (2018a) provide empirical evidence for the existence of a 3D-MFD at the urban scale based on data collected from the city of Zurich and London, respectively. Building on the two papers above, Loder et al. (2019) shows that the empirical 3D-MFD for both Zurich and London can be fitted using a specific functional form of the 3D-MFD for vehicle flow. Focusing on the passenger flow, Dakic et al. (2019a) develops an innovative method to estimate a passenger 3D-MFD model, which further demonstrates that the 3D-MFD model is well-defined for passenger flow and indeed exists empirically. Furthermore, Dakic et al. (2019b) shows that even if passenger occupancy cannot be dynamically estimated, as long as an appropriate occupancy of the modes is assumed, network control performance based on the 3D-MFD will not deviate significantly from the theoretical optimum. Hence, 3D-MFDs can be a useful monitoring tool for bimodal networks to develop network control strategies.

Appropriate infrastructure is another major consideration. To regulate the inflows of the two modes independently, dedicated lanes must exist around the perimeter of the network. Note that this does not mean dedicated lanes must exist upstream of all perimeter intersections at all times. Rather, a bimodal strategy can be achieved, as long as all public transport lines can travel on dedicated lanes (continuous or not) approaching the city center during the regulated period. This means that perimeter intersections without public transport lines do not need to have dedicated lanes. Also, all dedicated lanes only need to be enforced during peak periods. The existing road network infrastructure in many cities roughly satisfies such criteria. For example, in many European cities (such as Zurich), public transport is strongly promoted and almost all public transport lines access the city center through dedicated lanes. Another example will be many Chinese cities (such as Beijing), where the newly built infrastructure has typically 4-6 lanes per direction, hence almost all major roads accessing the city center have at least one lane dedicated to public transport. In these cities, a bimodal strategy can be implemented with proper signalization on the perimeter. This includes using traditional traffic signals, and other more sophisticated strategies such as flexible-sharing strategies (He et al., 2018, Yang et al., 2018a, 2019a).

This research aims to propose, model, and analyze a bimodal perimeter control strategy to provide public transport priority in the perimeter of urban networks, which is the first of this kind in literature to the best of our knowledge. In doing so, we first develop in Section 2 an integrated and systematic framework for the optimization of bimodal urban networks using 3D-MFDs, considering the complexities of bimodality. Then, in Section 3 , the bimodal perimeter control strategy is mathematically modelled. The complex interactions between the two modes both within the network and at the network perimeter are incorporated in the model. The bimodal strategy is then optimized using the methodological framework. Its performance at steadystates is analytically evaluated in Section 4 . We derive the theoretical lower bound for improvement when the bimodal strategy is properly implemented, compared to the single-mode strategy. Next, in Section 5 . we use macroscopic simulations to evaluate the performance of the bimodal strategy with realistic demands. The simulation model is based on the city center of Zurich and uses an empirical 3D-MFD for this area. Lastly, in Section 6, we test the sensitivity of the proposed strategy to variations in the traffic scenarios. Based on the results, Section 7 offers recommendations to the policy makers. 


\section{Methodological framework for bimodal urban networks using 3D-MFDs}

Table 1 summarizes the notation for the main variables and parameters in this paper.

A bimodal urban network consists of the public transport mode and the car mode. Denote the modes with $\xi \in\{b, c\}$, where $b$ is the public transport mode and $c$ is the car mode. The vehicle accumulation within the network is $\boldsymbol{n}=\left(n^{b}, n^{c}\right)^{T}$, where $n^{\xi}$ is the accumulation of the respective mode. We assume the network is homogeneous, i.e. both public transport vehicles and cars are generally uniformly distributed in the network. We assume further this homogeneous network has a well-defined 3D-MFD, which relates the average circulation flow of the two modes to the vehicle accumulation of the two modes in the network (Geroliminis et al., 2014, Ampountolas et al., 2017). This relationship can be affected by factors such as spatial configuration, space allocation between modes, public transport routes, etc. Recent empirical studies (Loder et al. 2017) have shown that such homogeneous bimodal networks do exist in reality, where there is a low scatter 3D-MFD. A 3D-MFD is a powerful tool not only to monitor a bimodal network (Loder et al. 2017), but also to optimize its planning, management and control. In this section, we aim to develop a methodological framework for the optimization of bimodal urban networks using 3D-MFDs.

Such a methodological framework is necessary due to the complexity of bimodal networks. While a singlemode network is often optimized at the accumulation with the highest flow (i.e. capacity), a bimodal network is more difficult to evaluate. This is because the performance of the two modes is often coupled (unless all lanes are dedicated), and the improvement of one mode can come at the expense of the other mode. Moreover, optimizing traffic flows is often not the only objective of the policy makers. Goals such as public transport priority are also important considerations, and different policy makers have different goals. Therefore, when considering a bimodal network, the two modes need to be analyzed with an integrated and systematic framework which incorporates these complexities and is generally applicable to different policy makers.

To this aim, denote the circulation flow with $\boldsymbol{q}=\left(q^{b}, q^{c}\right)^{T}$, where $q^{\xi}$ is the circulation flow of the respective mode. We represent the accumulation-flow relationship of this bimodal network with a vector function of the following form in this paper:

$$
\boldsymbol{q}(\boldsymbol{n})=\left(\begin{array}{l}
q^{b}\left(n^{b}, n^{c}\right) \\
q^{c}\left(n^{b}, n^{c}\right)
\end{array}\right)
$$

With this vector function representation, we propose to exploit the mathematical framework of a Pareto optimization to holistically evaluate bimodal urban networks. Pareto optimization is an important area of mathematics used for multiple-criteria decision making involving more than one objective function to be optimized simultaneously. In these cases, optimal decisions often need to be taken in the presence of trade-offs between two or more conflicting objectives. The optimization of bimodal networks is exactly such a case, hence Pareto optimization provides an applicable framework. In Eq, $1, q^{b}(\boldsymbol{n})$ and $q^{c}(\boldsymbol{n})$ can be considered as two objective functions of the optimization problem, where $\boldsymbol{n}$ belongs to the decision space $\mathcal{N}$. This decision space is bounded. For example, every network has a limited storing capability. In particular, the accumulation of cars, when public transport vehicles are not present, cannot exceed the jam density. Assuming storing each additional public transport vehicle comes at the expense of a fixed number of cars, $\mathcal{N}$ is a polygon with linear constraints. $\boldsymbol{q}$ is the objective vector and belongs to the objective space $\mathcal{Q}$. This objective space is also bounded, for example by the capacity of the network.

Evidently, we do not expect an accumulation $\boldsymbol{n}$ to optimize vehicle flows for both modes. Therefore, we exploit the concept of Pareto optimal. Mathematically, Pareto optimal solutions are those that cannot be improved in any of the objectives without degrading at least one of the other objectives. Similarly, we can define the Pareto accumulations for a bimodal network as those where the resulting circulation flow of one mode cannot be improved without reducing that of the other. The set of Pareto accumulations is then the 
Table 1: List of important variables

\begin{tabular}{|c|c|}
\hline \multicolumn{2}{|r|}{ Network level parameters: } \\
\hline$\xi \in\{b, c\}$ & $b$ is the public transport mode, $c$ is the car mode \\
\hline $\boldsymbol{n}=\left(n^{b}, n^{c}\right)^{T}$ & vehicle accumulation within the network $[\mathrm{veh}]^{*}$ \\
\hline $\mathcal{N}$ & decision space: the set of all feasible vehicle accumulations $\left(n^{b}, n^{c}\right)^{T}$ \\
\hline$\tilde{\boldsymbol{n}}=\left(\tilde{n}^{b}, \tilde{n}^{c}\right)^{T}$ & target vehicle accumulation at steady-state for the network [veh] \\
\hline $\boldsymbol{n}_{\text {out }}=\left(n_{\text {out }}^{b}, n_{\text {out }}^{c}\right)^{T}$ & $\begin{array}{l}\text { The accumulation of vehicles queueing in the perimeter to enter the network, i.e. the } \\
\text { queue at perimeter [veh] }\end{array}$ \\
\hline $\boldsymbol{q}=\left(q^{b}, q^{c}\right)^{T}$ & average circulating vehicle flow within the network $[\mathrm{veh} / \mathrm{h}]$ \\
\hline $\mathcal{Q}$ & objective space: the set of all feasible vehicle flows $\left(q^{b}, q^{c}\right)^{T}$ \\
\hline $\mathcal{G}(\cdot)$ & Pareto frontier of the bimodal network \\
\hline$w^{\xi}$ & weights of respective mode for a linear objective \\
\hline$p$ & average network passenger flow $[\mathrm{pax} / \mathrm{h}]$ \\
\hline$h^{\xi}$ & passenger occupancy of the respective mode [pax/veh] \\
\hline $\boldsymbol{d}_{\mathrm{ext}}=\left(d_{\mathrm{ext}}^{b}, d_{\mathrm{ext}}^{c}\right)^{T}$ & demand from outside the network with destinations inside the network $[\mathrm{veh} / \mathrm{h}]$ \\
\hline$r$ & car to public transport ratio of the incoming demand \\
\hline $\boldsymbol{d}_{\mathrm{int}}=\left(d_{\mathrm{int}}^{b}, d_{\mathrm{int}}^{c}\right)^{T}$ & $\begin{array}{l}\text { demand from within the network with destinations inside or outside the network, i.e. } \\
\text { internal demand }[\mathrm{veh} / \mathrm{h}]\end{array}$ \\
\hline $\boldsymbol{\mu}=\left(\mu^{b}, \mu^{c}\right)^{T}$ & inflow into the network $[\mathrm{veh} / \mathrm{h}]$ \\
\hline$\mu$ & total vehicle inflow into the network $[\mathrm{PCE} / \mathrm{h}]$ \\
\hline$\tilde{\boldsymbol{\mu}}=\left(\tilde{\mu}^{b}, \tilde{\mu}^{c}\right)^{T}$ & steady-state inflow at target vehicle accumulation into the network $[\mathrm{veh} / \mathrm{h}]$ \\
\hline$\tilde{\mu}$ & total vehicle inflow into the network at steady-state $[\mathrm{PCE} / \mathrm{h}]$ \\
\hline$\rho$ & passenger car equivalence (PCE) factor for a unit of public transport vehicle \\
\hline $\boldsymbol{\theta}=\left(\theta^{b}, \theta^{c}\right)^{T}$ & composition of the inflow traffic $\left(\rho \theta^{b}+\theta^{c}=1\right)$ \\
\hline $\boldsymbol{g}=\left(g^{b}, g^{c}\right)^{T}$ & trip completion rate within the network $[\mathrm{veh} / \mathrm{h}]$ \\
\hline$\eta^{\xi}$ & proportional factor for trip completion rate of the respective mode \\
\hline$Q$ & $\begin{array}{l}\text { upper bound for the total inflow into the network (network perimeter capacity) } \\
{[\mathrm{PCE} / \mathrm{h}]}\end{array}$ \\
\hline$Q^{\xi}$ & $\begin{array}{l}\text { upper bound for the total inflow on dedicated lanes of respective mode into the net- } \\
\text { work (network perimeter car/public transport capacity) }[\mathrm{PCE} / \mathrm{h}]\end{array}$ \\
\hline$Q^{f}$ & $\begin{array}{l}\text { upper bound for the total inflow into the perimeter intersections with flexible-sharing } \\
\text { strategies (network perimeter shared capacity) }[\mathrm{PCE} / \mathrm{h}]\end{array}$ \\
\hline
\end{tabular}

Intersection level parameters:

\begin{tabular}{l|l}
\hline$m \in M$ & intersections on the perimeter \\
\hline$i \in I, I \subseteq M$ & intersections on the perimeter with dedicated public transport lanes \\
\hline$j \in J, J \subseteq M$ & intersections on the perimeter with flexible-sharing strategies \\
\hline$l \in L, L \subseteq M$ & $\begin{array}{l}\text { intersections on the perimeter with mixed lanes (and no public transport lines going } \\
\text { into the network) }\end{array}$ \\
\hline $\boldsymbol{\mu}_{m}=\left(\mu_{m}^{b}, \mu_{m}^{c}\right)$ & inflow at intersection $m[\mathrm{veh}]$ \\
\hline$\mu_{m}$ & total inflow at intersection $m[\mathrm{PCE} / \mathrm{h}]$ \\
\hline$Q_{m}$ & upper bound for total inflow at intersection $m[\mathrm{PCE} / \mathrm{h}]$ \\
\hline$Q_{m}^{\xi}$ & upper bound for inflow of respective mode at intersection $m[\mathrm{PCE} / \mathrm{h}]$ \\
\hline$\beta_{j}$ & $\begin{array}{l}\text { linear reduction factor for capacity at intersection } j \text { when a flexible-sharing strategy } \\
\text { is implemented }\end{array}$ \\
\hline
\end{tabular}

* In this paper, $(\cdot)^{T}$ represents the transpose of a column vector. 
Pareto frontier of the bimodal network.

A bimodal network can then be evaluated by determining its Pareto frontier. Since there are infinite Pareto accumulations for any bimodal network, the optimization problem needs to be scalarized to offer a target accumulation for planning, management, and control purposes. Mathematically, scalarization means formulating a single-objective optimization problem such that optimal solutions to the single-objective optimization problem are also Pareto optimal solutions. We propose a linear scalarization approach by giving positive weights to $q^{b}$ and $q^{c}$. Since the appropriate weights can be determined by the policy makers depending on their priorities, such a methodology is generally applicable.

Definition 1. A linear objective of a bimodal network is one of the form $\max _{\boldsymbol{n} \in \mathcal{N}} w^{b} q^{b}(\boldsymbol{n})+w^{c} q^{c}(\boldsymbol{n})$, where $w^{b}$ and $w^{c}$ are positive weights.

An optimal target accumulation for a bimodal network can then be calculated by optimizing its linear objective defined by the policy maker. For example, following the treatment in previous studies (Geroliminis et al. 2014), the average passenger flow $p$ can be approximated with $p(\boldsymbol{n})=h^{b} q^{b}\left(n^{b}, n^{c}\right)+h^{c} q^{c}\left(n^{b}, n^{c}\right)$, where $h^{\xi}$ is the average passenger occupancy of the respective mode. This can be used as a linear objective of the bimodal network, which is sometimes also called the 3D-pMFD. This formulation assumes that the passenger occupancies have small variations over a reasonable time period (e.g. 30 minutes), and there is no instantaneous mode shift due to control strategies. In this paper, we will illustrate the general methodology using this weighting as an example. However, it should be noted that the methodology still applies with any specified weighting factors tailored to the goals of the policy makers.

We assume that the Pareto frontier is convex. This assumption is consistent with the findings from empirical studies (Loder et al., 2017) and simulations (Geroliminis et al., 2014; Ortigosa et al., 2017; Dakic and Menendez, 2018). For example, the empirical 3D-MFD fitted with data collected from the city center of Zurich (Loder et al., 2017) can be represented by Eq.2.

$$
\boldsymbol{q}(\boldsymbol{n})=\left(\begin{array}{c}
0.3769 n^{b}-0.00002519 n^{b} n^{c}-0.0005679\left(n^{b}\right)^{2} \\
0.7162 n^{c}-0.004268 n^{b} n^{c}-0.0001893\left(n^{c}\right)^{2}
\end{array}\right)
$$

$q^{b}(\boldsymbol{n})$ and $q^{c}(\boldsymbol{n})$ are plotted in Figure $1 \mathrm{a}$ and b, respectively, for the bimodal network optimization problem. The shape of $q^{b}(\boldsymbol{n})$ and $q^{c}(\boldsymbol{n})$ is similar to that derived from previous simulation studies (Geroliminis et al., 2014; Ortigosa et al., 2017). Therefore, it is reasonable to assume that its qualitative properties are reproducible in a general 3D-MFD. The objective space $\mathcal{Q}$ for the city center of Zurich and the Pareto frontier are plotted in Figure 17. Note that the Pareto frontier is convex. Since the Pareto frontier calculated from the 3D-MFD of previous simulation studies is also convex, it is reasonable to assume that a general Pareto frontier is convex. Figure $1 \mathrm{~d}$ presents the linear objective using a 3D-pMFD, assuming an average passenger occupancy $h^{b}=55 \mathrm{pax} /$ veh and $h^{c}=1.2 \mathrm{pax} /$ veh. Note that the optimal solution occurs at some non-zero public transport accumulation, which is verified to be a Pareto accumulation. This is because the higher passenger occupancy of public transport makes it a more efficient mode than the car mode from a passenger perspective. Additionally, observe that the optimum car accumulation is lower when the network has a higher public transport accumulation. Therefore, adding public transport to the network increases the network efficiency by using the limited road space to move more people, but it also makes the network more prone to congestion at a lower car demand.

Note that Eq.2 is only to illustrate the form of the 3D-MFD. The analytical modeling and results presented in Sections 2 and Section 3 do not depend on this specific functional form, but instead use the general form of the 3D-MFD as shown in Eq.1. We will come back to Eq2 in Section 5 when we use simulations to test the analytical results. 

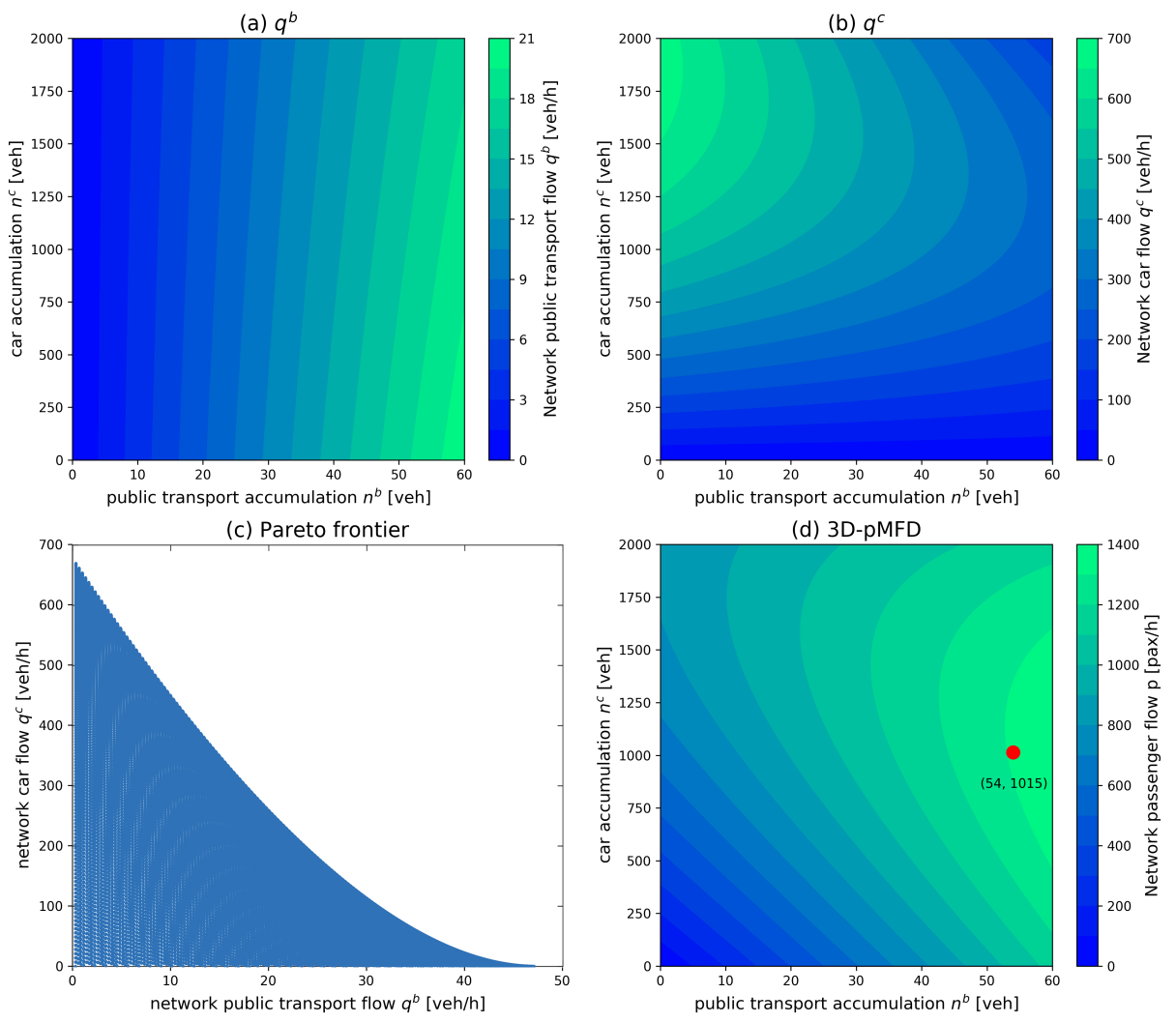

Figure 1: The city center of Zurich: a. Public transport flow vs. accumulations. b. Car flow vs. accumulations. c. The objective space and Pareto frontier. d. Linear objective using 3D-pMFD assuming an average passenger occupancy $h^{b}=55 \mathrm{pax} / \mathrm{veh}$ and $h^{c}=1.2 \mathrm{pax} / \mathrm{veh}$. The identified dot is the optimal solution.

\section{Bimodal perimeter control strategy}

With the proposed methodological framework for bimodal networks, effective strategies can be designed to meet the planning, management, and control goals of policy makers. In particular, strategies to provide public transport priority on the network level can be evaluated in an integrated and systematic manner incorporating the complexities of bimodal networks. In the rest of the paper, we propose, model, and analyze one such strategy, a bimodal perimeter control strategy, as an application of our methodological framework. To the authors' best knowledge, this is the first perimeter control strategy which provides public transport priority in the perimeter the urban networks. In this section, we will develop a mathematical model of this bimodal strategy.

We consider the problem of commute into a bimodal urban network during the peak period. During this period, there is often some vehicles queueing in the perimeter to enter the network. Denote this with $\boldsymbol{n}_{\text {out }}=\left(n_{\text {out }}^{b}, n_{\text {out }}^{c}\right)^{T}$, where $n_{\text {out }}^{\xi}$ is the queue at the perimeter of the respective mode. We assume that before the morning peak period, the accumulation $n^{\xi}$ within the network is small, and the queue at perimeter $n_{\text {out }}^{\xi}$ is zero. During the peak period, there is a strong demand $\boldsymbol{d}_{\text {ext }}=\left(d_{\text {ext }}^{b}, d_{\text {ext }}^{c}\right)^{T}$ going into the network, where $d_{\mathrm{ext}}^{\xi}$ is this external demand of the respective mode. Evidently, there is also some internal demand $\boldsymbol{d}_{\mathrm{int}}=\left(d_{\mathrm{int}}^{b}, d_{\mathrm{int}}^{c}\right)^{T}$ originated from within the network, where $d_{\mathrm{int}}^{\xi}$ is this internal demand of the respective mode. During the morning peak periods, it is reasonable to assume the network operation is dominantly 
influenced by the incoming demand $d_{\text {ext }}^{\xi}$ (i.e. $d_{\text {ext }}^{\xi}$ is much larger than $d_{\text {int }}^{\xi}$ ). As reviewed in the introduction, previous works on perimeter control have shown that, it is beneficial to the system to limit the inflow and leave some vehicles queued outside the network, when the network is subject to a very large incoming demand. This helps to maintain the network flow near the maximum value of the MFD. In contrast, if the inflow is not controlled at the perimeter, the traffic within the network can quickly become congested. Therefore, the total delay incurred in the system (considering both outside of and within the network) during the peak period can be larger without perimeter control.

As elaborated in the introduction, none of the existing perimeter control strategies developed so far have preferential treatment for different modes. In particular, they are not able to regulate the inflows of public transport vehicles and cars independently, but only the total vehicle flow, i.e. the two inflows are dependent. We propose a bimodal perimeter control strategy for bimodal networks to provide public transport priority in the network perimeter. In the bimodal strategy, the inflows of public transport vehicles and cars can be regulated independently (i.e. both inflows are controllable). Denote the inflow into the network with a vector $\boldsymbol{\mu}=\left(\mu^{b}, \mu^{c}\right)^{T}$, where $\mu^{\xi}$ is the inflow of the respective mode. If the passenger car equivalence factor (PCE) for a unit of public transport vehicle is $\rho$, the total network inflow in PCE $/ \mathrm{h}$ is $\mu=\rho \mu^{b}+\mu^{c}$. Mathematically, all previous models on perimeter control strategies only control this total inflow $\mu \in \mathbb{R}$ without considering different modes. In contrast, our control parameter is a two dimensional vector $\boldsymbol{\mu} \in \mathbb{R}^{1 \times 2}$ which controls the two modes independently.

The dynamics of the bimodal network where the inflows of both modes can be regulated independently (i.e. applying a bimodal strategy) can be described by the following ordinary differential equation:

$$
\dot{\boldsymbol{n}}(t)=\boldsymbol{d}_{\mathrm{int}}(t)-\boldsymbol{g}(\boldsymbol{n}(t))+\boldsymbol{\mu}(t)
$$

$\boldsymbol{n}=\left(n^{b}, n^{c}\right)^{T}$ is the network accumulation. $\dot{\boldsymbol{n}}(t)$ is the change in network accumulation over time. $\boldsymbol{d}_{\text {int }}=$ $\left(d_{\text {int }}^{b}, d_{\text {int }}^{c}\right)^{T}$ is the traffic generation from within the network, which is considered as a control disturbance in our model. $\boldsymbol{g}=\left(g^{b}, g^{c}\right)^{T}$ is the trip completion rate within the network, where $g^{\xi}$ is the rate of the respective mode. Following Geroliminis and Daganzo (2008), $g^{\xi}$ is assumed to be proportional to $q^{\xi}$ with a factor $\eta^{\xi}$ for each mode, i.e. $g^{\xi}=\eta^{\xi} q^{\xi}$. This factor is a property of the network and can be calculated empirically or with simulations. $\boldsymbol{\mu}=\left(\mu^{b}, \mu^{c}\right)^{T}$ is the inflow into the network, which is the control parameter in our model. Note that all variables in $\mathrm{Eq} 3$ are time-dependent two-dimensional vectors.

In contrast, if only the total inflow can be controlled for the bimodal network (i.e. single-mode control), the control parameter $\boldsymbol{\mu}$ is subject to the additional constraint:

$$
\boldsymbol{\mu}(t)=\mu(t) \boldsymbol{\theta}(t)
$$

$\boldsymbol{\theta}=\left(\theta^{b}, \theta^{c}\right)^{T}$ describes the composition of the inflow traffic, i,e, $\mu^{c} / \mu^{b}=\theta^{c} / \theta^{b}$. Denote this ratio with $r$. One can verify that $\rho \theta^{b}+\theta^{c}=1$. Note that the control parameter in this case is only the total inflow $\mu$, hence there is one fewer degree of freedom in the solution. The mode composition $\boldsymbol{\theta}$ is determined by the composition of the incoming demand or queued vehicles in the perimeter, hence it cannot be controlled and is an input variable. Since single-mode strategies need to satisfy this additional constraint, we expect to find more efficient control solutions by regulating the inflows of the two modes independently with the bimodal strategy.

Perimeter control strategies usually aim to maintain the network in a steady-state at a target accumulation. However, this steady-state can only be realized if there is enough incoming demand arriving at the perimeter. For the single-mode strategy, this is usually not a problem, because $d_{\text {ext }}^{c}$ is high enough during the peak period, otherwise perimeter control would not be necessary in the first place. For the bimodal strategy, this leads to a potential problem. In many urban areas, there are fewer public transport vehicles in the network 
than the optimal amount. Although theoretically, the public transport agency can increase the public transport frequency, in practice this is often not possible due to the operation costs, the fixed schedules, etc. Therefore, it is very likely that the calculated bus inflow cannot be realized due to inadequate incoming public transport $d_{\mathrm{ext}}^{b}$. Moreover, recall from Figure $1 \mathrm{~b}$ that when the system targets a higher public transport accumulation, the corresponding optimal car accumulation will be lower. Therefore, if a very high public transport accumulation is unattainable, the perimeter control strategy will let in fewer cars than the actual optimum amount corresponding to the attained public transport accumulation. This reduces the efficiency of the perimeter control. To completely resolve this problem, the real time incoming public transport demand $d_{\mathrm{ext}}^{b}(t)$ can be imposed as a time-varying constraint. However, in practice, accurate real time information is often not available, and only a planned public transport schedule might be available. Hence, to reduce the data requirements and the computational complexity, we propose to solve this problem heuristically by treating the incoming public transport demand $d_{\text {ext }}^{b}(t)$ as a step-function constraint, i.e. it is assumed to be constant for some period (e.g. 30 minutes). This is a reasonable assumption because although there are inevitable deviations from the planned schedule, for example due to bus bunching, the average headway over a long enough period of time should be reasonably close to the service plan. Overall, the optimal control solution at steady-state needs to satisfy the constraint $\mathbf{0} \leq \boldsymbol{\mu} \leq \boldsymbol{d}_{\text {ext }}$.

Adopting our methodological framework, the target accumulation at steady-state for a general linear objective, denoted with $\tilde{\boldsymbol{n}}=\left(\tilde{n^{b}}, \tilde{n}^{c}\right)^{T}$, can be solved from the optimization problem:

$$
\max _{\boldsymbol{n}} p(\boldsymbol{n})=w^{b} q^{b}(\boldsymbol{n})+w^{c} q^{c}(\boldsymbol{n})
$$

subject to

$$
\left\{\begin{array}{l}
\boldsymbol{d}_{\mathrm{int}}-\boldsymbol{g}+\boldsymbol{\mu}=0 \\
\mathbf{0} \leq \boldsymbol{\mu} \leq \boldsymbol{d}_{\mathrm{ext}}
\end{array}\right.
$$

Another important consideration for the bimodal perimeter strategy is the infrastructure at the perimeter implemented to provide bus priority. For the single-mode strategy, it is only required that the car inflows at all perimeter intersections must be regulated, for example with traffic signals. For the bimodal strategy, to independently regulate the inflows of the two modes, we assume all public transport vehicles can jump the car queues upstream of the perimeter intersections during the peak period, for example by entering the controlled area through dedicated lanes. We consider this a fair assumption. On one hand, such infrastructure does exist in some cities around the world, as described in the introduction. On the other hand, when considering any perimeter control strategies for a bimodal network, it is arguable whether the strategy can be approved in practice at all, if such infrastructure does not already exist. If public transport is queued outside of the network among the cars due to lack of proper infrastructure, the perimeter control strategy might meet with strong opposition from involved stakeholders such as public transport users and agencies, even if the traffic operation within the network might be improved for both modes.

An intuitive choice to realize independent regulation of the two modes is to implement dedicated lanes coming in up to the perimeter intersections and apply separate signals to public transport and cars. This, however, comes with the expense of reduced capacities at these intersections. Although the capacities of the perimeter intersections do not impose constraints to the network inflows at steady states (otherwise perimeter control strategies would not be necessary), it can impose constraints during transient periods. This can harm the overall traffic performance, because cars might not be discharged fast enough in the perimeter during transient periods to reach the target accumulation for optimal operation inside the network. Meanwhile, cars might be unnecessarily queued outside of the network due to limited discharge capacity. Therefore, the traffic operation of the entire network, both inside and outside of the perimeter, can be harmed.

To overcome this problem, a class of public transport priority strategies called flexible-sharing strategies 
has been recently analyzed by He et al. (2018). These strategies share road space capacity in a flexible manner at a given element (e.g. the intersection) to move public transport vehicles in front of the car queues without continuously banning cars from using one full lane. Such flexible space allocation combines the advantages of mixed lanes (the element's capacity can be fully utilized if public transport vehicle flow is low) and dedicated lanes (public transport receives priority). This is especially relevant to the bimodal perimeter control strategy, where the inflow of each mode needs to be regulated independently while the perimeter capacity can be fully utilized when necessary. A specific example of flexible-sharing strategies, the pre-signals, will be illustrated and discussed in Section 5 .

Without looking at specific infrastructure configurations, we model how different infrastructure types (i.e. mixed lanes, dedicated lanes, and flexible sharing strategies) in the network perimeter will result in different constraints on the inflow during transient periods. The inflow capacity of the network perimeter depends on many factors. Some of these factors, e.g. intersection configuration and capacity of the links, are fixed for a given network. These factors determine the upper bound $Q$ for the total inflow $\mu$ into the network. However, the actual discharge capacity of the network, which depends on factors such as the green time ratio and the percentage of through-going traffic, can be smaller than this upper bound. Since perimeter control strategies regulate the perimeter inflows by controlling the green time ratio, the actual discharge capacity changes over time. When we refer to the capacity of the network perimeter in the rest of the paper, we are referring to the upper bound $Q$ for the given network, rather than the instantaneous discharge capacity.

The capacity of the network perimeter can be reserved for each mode with dedicated lanes, or shared by the two modes with mixed lanes and flexible-sharing strategies. This determines constraints to the network inflows. Index the intersections on the perimeter with $m \in M$. Further index those with dedicated public transport lanes for the incoming approach with $i, i \in I, I \subseteq M$, and those with flexible-sharing strategies for the incoming approach with $j, j \in J, J \subseteq M$. We assume for perimeter intersections with incoming public transport lines, either dedicated public transport lanes or flexible-sharing strategies are implemented on the incoming approach. Index the perimeter intersections without incoming public transport lines, with $l, l \in L$, $L \subseteq M$. Overall, the intersection sets $I, J$, and $L$ are mutually exclusive and collectively exhaustive.

The aggregate perimeter constraints on the total inflow is determined by the individual constraints on the inflow at each individual intersection. Denote the inflow at intersection $m$ with $\boldsymbol{\mu}_{m}=\left(\mu_{m}^{b}, \mu_{m}^{c}\right)$, where $\mu_{m}^{\xi}$ is the inflow for the respective mode. We have $\mu_{m}=\rho \mu_{m}^{b}+\mu_{m}^{c}$ and $\mu^{\xi}=\sum_{m} \mu_{m}^{\xi}$. Denote the upper bound of $\mu_{m}, \mu_{m}^{b}$, and $\mu_{m}^{c}$ at each individual intersection respectively with $Q_{m}, Q_{m}^{b}$, and $Q_{m}^{c}$. We have $Q_{i}=Q_{i}^{b}+Q_{i}^{c}$, $Q_{j}=Q_{j}^{c}$, and $Q_{l}=Q_{l}^{c}$. Further denote the upper bounds of $\sum_{m \in I \cup L} \mu_{m}^{c}, \sum_{m \in I} \mu_{m}^{b}$, and $\sum_{m \in J} \mu_{m}$ respectively with $Q^{c}, Q^{b}$, and $Q^{f}$, and refer to them in the rest of the paper respectively as the network perimeter car capacity, the network perimeter public transport capacity, and the network perimeter shared capacity. We have,

$$
Q=Q^{b}+Q^{c}+Q^{f}
$$

Note that we do not discuss in this paper how to calculate $\mu_{m}^{\xi}$, i.e. how the total inflow $\mu^{\xi}$ should be distributed among the individual perimeter intersections. Evidently, this depends on the specific network considered and the traffic management and control goals. Some of the physical parameters to consider include the spatial distribution of the incoming demand over the network perimeter, the capacities of each perimeter intersection, and the link storage capacity upstream of each perimeter intersection. Optimization techniques such as the model predictive approach proposed in Yang et al. (2018b) can be used to calculate $\mu_{m}^{\xi}$ for a given network. This paper models and analyzes the proposed bimodal strategy at a aggregate level. 


\section{Performance of the bimodal perimeter control strategy at steady-states}

In this section, we analytically evaluate the performance of a properly implemented bimodal perimeter control strategy at steady-states using the methodological framework proposed in Section 2 and the strategy model developed in Section 3. In particular, we compare its performance to the case when a single-mode strategy is properly implemented. The purpose is to analyze the improvement in passenger flow (Eq $\sqrt[5]{5}$, and derive the theoretical lower bound for improvement.

We denote the Pareto frontier of the network with a general convex function $\mathcal{G}(\cdot)$, where we have $q^{c} \leq \mathcal{G}\left(q^{b}\right)$. A properly implemented strategy should optimize the network operation at steady-states. In our case, we aim to optimize the average passenger flow as a linear objective. Therefore, we aim to solve the optimization problem presented in Eq 5 and 6 , choosing the passenger occupancies as weights for the linear objective. This is not easy, because the 3D-MFD (Eq. 1) is non-linear, as is the case in Eq. 2. Therefore, we wish to eliminate the non-linear terms, i.e. $\boldsymbol{q}$ and $\boldsymbol{g}$, from Eq.5 and Eq 6 . Notice that $q^{\xi}=g^{\xi} / \eta^{\xi}, \xi \in\{b, c\}$. From Eq, we have $\boldsymbol{g}=\boldsymbol{d}_{\text {int }}+\boldsymbol{\mu}$ satisfying $g^{c} / \eta^{c} \leq \mathcal{G}\left(g^{b} / \eta^{b}\right)$. Accordingly, we can replace $\boldsymbol{g}$ and $\boldsymbol{q}$ in the optimization problem, and rewrite Eq 5 and 6 as an optimization for a function $P$ of decision variable $\boldsymbol{\mu}$.

$$
\max _{\boldsymbol{\mu}} P(\boldsymbol{\mu})=h^{b} \mu^{b} / \eta^{b}+h^{c} \mu^{c} / \eta^{c}
$$

subject to

$$
\left\{\begin{array}{l}
\left(\mu^{c}+d_{\mathrm{int}}^{c}\right) / \eta^{c} \leq \mathcal{G}\left(\left(\mu^{b}+d_{\mathrm{int}}^{b}\right) / \eta^{b}\right) \\
\mathbf{0} \leq \boldsymbol{\mu} \leq \boldsymbol{d}_{\mathrm{ext}}
\end{array}\right.
$$

For the single-mode strategy, recall from Eq, 4 that the car to public transport ratio $\theta^{c} / \theta^{b}$ of the inflow traffic cannot be controlled. This imposes an additional constraint on $\boldsymbol{\mu}$ for the target steady-state to be realizable, i.e.

$$
\mu^{c}=r \mu^{b}
$$

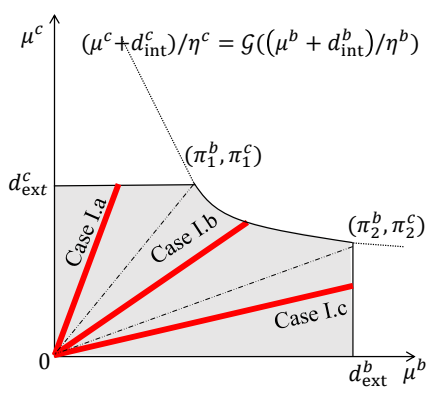

(a) Case I: $\pi_{1}^{b}<\pi_{2}^{b}$

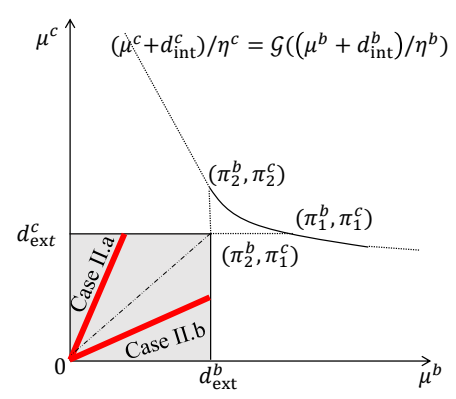

(b) Case II: $\pi_{1}^{b} \geq \pi_{2}^{b}$

Figure 2: Illustration of the feasible regions for both strategies. The feasible region for the single-mode strategy is the red bold line segment with slope $r$, whereas the feasible region for the bimodal strategy is the light gray region.

Therefore the constraints for $\boldsymbol{\mu}$ for both strategies can be illustrated as the feasible regions on the $\mu^{b}-\mu^{c}$ plane in Figure 2. We wish to evaluate the performance of properly implemented strategies by optimizing each strategy based on its respective feasible region. There are many different cases how their feasible regions can be, depending on the demands and the network characteristic (i.e. the Pareto frontier). 
Denote $\left(\pi_{1}^{b}, \pi_{1}^{c}\right)$ as the intersection between line $\mu^{c}=d_{\text {ext }}^{c}$ and curve $\left(\mu^{c}+d_{\text {int }}^{c}\right) / \eta^{c}=\mathcal{G}\left(\left(\mu^{b}+d_{\text {int }}^{b}\right) / \eta^{b}\right)$, and $\left(\pi_{2}^{b}, \pi_{2}^{c}\right)$ as the intersection between line $\mu^{b}=d_{\text {ext }}^{b}$ and curve $\left(\mu^{c}+d_{\text {int }}^{c}\right) / \eta^{c}=\mathcal{G}\left(\left(\mu^{b}+d_{\text {int }}^{b}\right) / \eta^{b}\right)$. Here it holds naturally that $\pi_{1}^{c}=d_{\mathrm{ext}}^{c}$ and $\pi_{2}^{b}=d_{\mathrm{ext}}^{b}$. Note that in the case where line $\mu^{c}=d_{\mathrm{ext}}^{c}$ does not intersect with curve $\left(\mu^{c}+d_{\text {int }}^{c}\right) / \eta^{c}=\mathcal{G}\left(\left(\mu^{b}+d_{\text {int }}^{b}\right) / \eta^{b}\right)$, denote $\left(\pi_{1}^{b}, \pi_{1}^{c}\right)=\left(0, \mathcal{G}\left(d_{\text {int }}^{b} / \eta^{b}\right)-d_{\text {int }}^{c}\right)$; and in the case where line $\mu^{b}=d_{\text {ext }}^{b}$ does not intersect with curve $\left(\mu^{c}+d_{\text {int }}^{c}\right) / \eta^{c} \leq \mathcal{G}\left(\left(\mu^{b}+d_{\text {int }}^{b}\right) / \eta^{b}\right)$, denote $\left(\pi_{2}^{b}, \pi_{2}^{c}\right)=\left(\mathcal{G}^{-1}\left(d_{\mathrm{int}}^{c} / \eta^{c}\right)-d_{\mathrm{int}}^{b} / \eta^{b}, 0\right)$, where $\mathcal{G}^{-1}$ represents the inverse function of $\mathcal{G}$.

Figure 2 shows two cases: $\pi_{1}^{b}<\pi_{2}^{b}$ (Case I, illustrated in Figure 2a) and $\pi_{1}^{b} \geq \pi_{2}^{b}$ (Case II, illustrated in Figure 2b). For Case I, there are three subcases based on the value of the car to public transport ratio $\mu^{c} / \mu^{b}=r$, i.e. Case I.a $\left(r>\pi_{1}^{c} / \pi_{1}^{b}\right)$, Case I.b $\left(\pi_{2}^{c} / \pi_{2}^{b}<r \leq \pi_{1}^{c} / \pi_{1}^{b}\right)$ and Case I.c $\left(r \leq \pi_{2}^{c} / \pi_{2}^{b}\right)$. These three cases correspond to three different feasible regions for the single-mode strategy. The boundaries between the feasible regions are shown in the dotted lines in Figure 2a). Similarly, for Case II, we have two subcases: Case II.a $\left(r>\pi_{1}^{c} / \pi_{2}^{b}\right)$ and Case II.b $\left(r \leq \pi_{1}^{c} / \pi_{2}^{b}\right)$.

With the definition of all cases, the results for this section can be summarized in Theorem 1 followed by one numerical example and the proof.

Theorem 1. 1) The theoretical lower bound for improvement in average passenger flow by properly implementing the bimodal strategy compared to the single-mode strategy is summarized in Table 2 .

Table 2: Improvement in the average passenger flow by properly implementing the bimodal strategy compared to the single-mode strategy $(\operatorname{pax} / \mathrm{h})$.

\begin{tabular}{|c|c|c|c|}
\hline & & $\frac{h^{b} \eta^{c}}{h^{c} \eta^{b}}>-\frac{\pi_{1}^{c}-\pi_{2}^{c}}{\pi_{1}^{b}-\pi_{2}^{b}}$ & $\frac{h^{b} \eta^{c}}{h^{c} \eta^{b}} \leq-\frac{\pi_{1}^{c}-\pi_{2}^{c}}{\pi_{1}^{b}-\pi_{2}^{b}}$ \\
\hline \multirow{3}{*}{$\begin{array}{l}\text { Case I: } \\
\pi_{1}^{b}<\pi_{2}^{b}\end{array}$} & $\begin{array}{l}\text { Case I.a: } \\
r>\pi_{1}^{c} / \pi_{1}^{b}\end{array}$ & $\frac{h^{b}\left(\pi_{2}^{b}-\pi_{1}^{c} / r\right)}{\eta^{b}}+\frac{h^{c}\left(\pi_{2}^{c}-\pi_{1}^{c}\right)}{\eta^{c}}$ & $\frac{h^{c}\left(r \pi_{1}^{b}-\pi_{1}^{c}\right)}{r \eta^{c}}$ \\
\hline & $\begin{array}{c}\text { Case I.b: } \\
\pi_{2}^{c} / \pi_{2}^{b}<r \leq \pi_{1}^{c} / \pi_{1}^{b}\end{array}$ & $\frac{h^{b}\left(\pi_{2}^{b}-\pi_{1}^{b}\right) / \eta^{b}+h^{c}\left(\pi_{2}^{c}-\pi_{1}^{c}\right) / \eta^{c}}{\left(r \pi_{2}^{b}-r \pi_{1}^{b}-\pi_{2}^{c}+\pi_{1}^{c}\right) /\left(-\pi_{2}^{c}+r \pi_{2}^{b}\right)}$ & $\frac{h^{b}\left(\pi_{1}^{b}-\pi_{2}^{b}\right) / \eta^{b}+h^{c}\left(\pi_{1}^{c}-\pi_{2}^{c}\right) / \eta^{c}}{\left(r \pi_{2}^{b}-r \pi_{1}^{b}-\pi_{2}^{c}+\pi_{1}^{c}\right) /\left(\pi_{1}^{c}-r \pi_{1}^{b}\right)}$ \\
\hline & $\begin{array}{l}\text { Case I.c: } \\
r \leq \pi_{2}^{c} / \pi_{2}^{b}\end{array}$ & $\frac{h^{c}\left(\pi_{2}^{c}-r \pi_{2}^{b}\right)}{\eta^{c}}$ & $\frac{h^{b}\left(\pi_{1}^{b}-\pi_{2}^{b}\right)}{\eta^{b}}+\frac{h^{c}\left(\pi_{1}^{c}-r \pi_{2}^{b}\right)}{\eta^{c}}$ \\
\hline \multirow{2}{*}{$\begin{array}{l}\text { Case II: } \\
\pi_{1}^{b} \geq \pi_{2}^{b}\end{array}$} & $\begin{array}{l}\text { Case II.a: } \\
r>\pi_{1}^{c} / \pi_{2}^{b}\end{array}$ & \multicolumn{2}{|c|}{$\frac{h^{b}\left(\pi_{2}^{b}-\pi_{1}^{c} / r\right)}{\eta^{b}}$} \\
\hline & $\begin{array}{l}\text { Case II.b: } \\
r \leq \pi_{1}^{c} / \pi_{2}^{b}\end{array}$ & \multicolumn{2}{|c|}{$\frac{h^{c}\left(\pi_{1}^{c}-r \pi_{2}^{b}\right)}{\eta^{c}}$} \\
\hline
\end{tabular}

2) Properly implementing the bimodal strategy can improve the passenger mobility of the network at steadystates.

Remark. To illustrate typical values obtained using the theoretical formulas given in Table 3 , we calculate some numerical values assuming a typical incoming public transport demand of $150 \mathrm{veh} / \mathrm{h}$ and passenger occupancy of 50pax/veh for the city center of Zurich, with empirical 3D-MFD given by Eq2 2 and depicted in Fig. 1. A range of incoming car demands and car to public transport ratios are tested. The infinite ratio represents the cases without any incoming public transport vehicles. The results are summarized in Table 3. This shows that properly implementing the bimodal strategy often results in sustainable improvement in the passenger mobility of the network at steady-states.

Proof for Theorem 1. 1) Note that the only difference between Eq 5 and Eq 8 is an additive constant term, therefore the improvement in the average passenger flow by adopting the bimodal strategy can be represented 
Table 3: Typical values for improvement (in pax/h and percentage) by properly implementing the bimodal strategy compared to the single-mode strategy according to theoretical formulas given in Table 3

\begin{tabular}{lcccc}
\hline & $r=50$ & $r=150$ & $r=500$ & $r=\infty$ \\
\hline$d_{\text {ext }}^{c}=10000 \mathrm{veh} / \mathrm{hr}$ & $120(15 \%)$ & $167(21 \%)$ & $260(33 \%)$ & $300(38 \%)$ \\
\hline$d_{\text {ext }}^{c}=15000 \mathrm{veh} / \mathrm{hr}$ & $257(28 \%)$ & $28(3 \%)$ & $137(15 \%)$ & $197(22 \%)$ \\
\hline$d_{\text {ext }}^{c}=20000 \mathrm{veh} / \mathrm{hr}$ & $257(28 \%)$ & $38(4 \%)$ & $116(13 \%)$ & $105(11 \%)$ \\
\hline
\end{tabular}

as

$$
\Delta p=P_{\mathrm{bi}}^{*}-P_{\text {single }}^{*}
$$

where $P_{\mathrm{bi}}^{*}$ and $P_{\text {single }}^{*}$ represent the optimal objective value Eq. 8 for bimodal and single-mode control, respectively.

Since the Pareto frontier $\mathcal{G}(\cdot)$ is convex, and the objective function $P(\boldsymbol{\mu})$ is linear, the optimal steady-state solution for the bimodal strategy lies in one of the vertices of the feasible region. Further notice that the objective function $P(\boldsymbol{\mu})$ has a negative slope, hence in Case I is $\left(\pi_{2}^{b}, \pi_{2}^{c}\right)$ if $\frac{h^{b} \eta^{c}}{h^{c} \eta^{b}}>-\frac{\pi_{1}^{c}-\pi_{2}^{c}}{\pi_{1}^{b}-\pi_{2}^{b}}$, and $\left(\pi_{1}^{b}, \pi_{1}^{c}\right)$ if $\frac{h^{b} \eta^{c}}{h^{c} \eta^{b}} \leq-\frac{\pi_{1}^{c}-\pi_{2}^{c}}{\pi_{1}^{b}-\pi_{2}^{b}}$. The optimal steady-state solution for the bimodal strategy in Case II is always $\left(\pi_{2}^{b}, \pi_{1}^{c}\right)$. An illustration example for Case I if $\frac{h^{b} \eta^{c}}{h^{c} \eta^{b}}>-\frac{\pi_{1}^{c}-\pi_{2}^{c}}{\pi_{1}^{b}-\pi_{2}^{b}}$ is shown in Figure 3

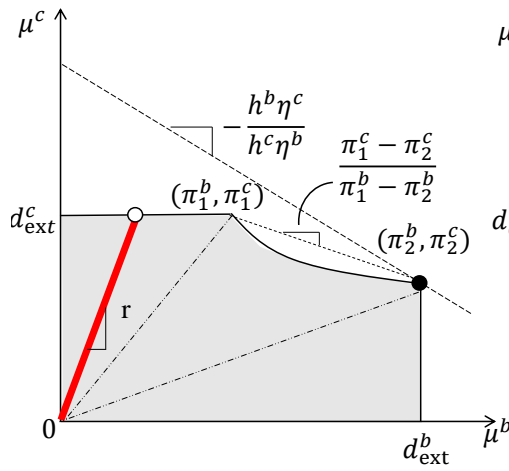

(a) Case I.a: $r>\pi_{1}^{c} / \pi_{1}^{b}$

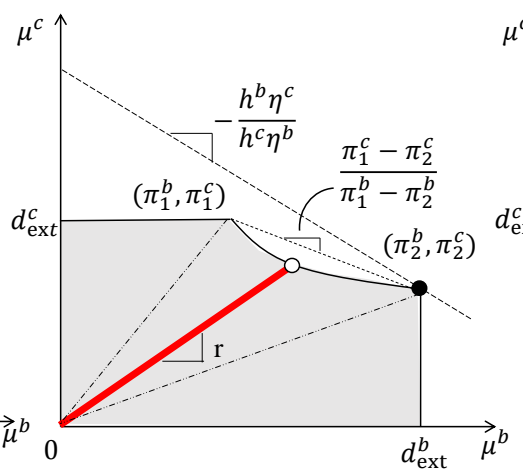

(b) Case I.b: $\pi_{2}^{c} / \pi_{2}^{b}<r \leq \pi_{1}^{c} / \pi_{1}^{b}$

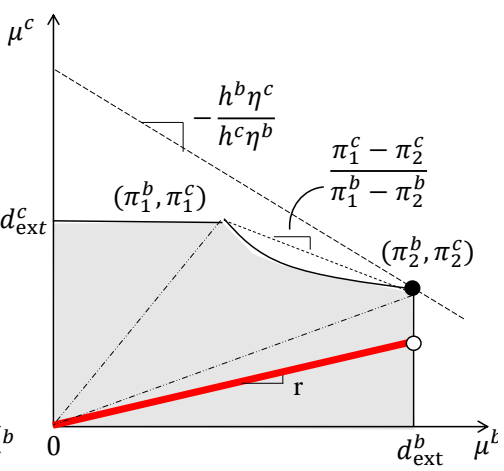

(c) Case I.c: $r \leq \pi_{2}^{c} / \pi_{2}^{b}$

Figure 3: Illustration of the feasible regions and objective function for both strategies. The feasible region for the single-mode strategy is the red bold line segment, whereas the feasible region for the bimodal strategy is the gray region. The objective function is represented by the dotted line, with a slope of $-\frac{h^{c} \eta^{b}}{h^{b} \eta^{c}}$.

For presentation simplicity, we focus on the Case I in the case $\frac{h^{b} \eta^{c}}{h^{c} \eta^{b}}>-\frac{\pi_{1}^{c}-\pi_{2}^{c}}{\pi_{1}^{b}-\pi_{2}^{b}}$.

In Case I, the objective function is always optimized at $\left(\pi_{2}^{b}, \pi_{2}^{c}\right)$ for the bimodal strategy,

$$
P_{\mathrm{bi}}^{*}=P\left(\pi_{2}^{b}, \pi_{2}^{c}\right)=\frac{h^{b} \pi_{2}^{b}}{\eta^{b}}+\frac{h^{c} \pi_{2}^{c}}{\eta^{c}}
$$

Next, we calculate the optimal solution for the single-mode strategy in the three subcases. The objective function and the feasible regions for the three cases are shown in Figure 3. Notice that the optimal solution 
for the single-mode strategy is always the non-zero vertex of the line segment representing the feasible region of the single-mode strategy, since the objective values for the single-mode strategy is always non-negative across the entire feasible region. The calculation of this vertex differs in the three subcases.

For Case I.a, i.e. $r>\pi_{1}^{c} / \pi_{1}^{b}$, the objective function is optimized at the non-zero vertex $\left(\pi_{1}^{c} / r, \pi_{1}^{c}\right)$ for the single-mode strategy,

$$
P_{\text {single }}^{*}=P\left(\pi_{1}^{c} / r, \pi_{1}^{c}\right)=\frac{h^{b} \pi_{1}^{c}}{r \eta^{b}}+\frac{h^{c} \pi_{1}^{c}}{\eta^{c}}
$$

Hence, the improvement is

$$
\Delta p=P_{\mathrm{bi}}^{*}-P_{\text {single }}^{*}=\frac{h^{b}\left(\pi_{2}^{b}-\pi_{1}^{c} / r\right)}{\eta^{b}}+\frac{h^{c}\left(\pi_{2}^{c}-\pi_{1}^{c}\right)}{\eta^{c}}
$$

For Case I.b, i.e. $\pi_{2}^{c} / \pi_{2}^{b}<r \leq \pi_{1}^{c} / \pi_{1}^{b}$, the objective function is optimized at the non-zero vertex, i.e. the intersection between the curve $\left(\mu^{c}+d_{\text {int }}^{c}\right) / \eta^{c} \leq \mathcal{G}\left(\left(\mu^{b}+d_{\text {int }}^{b}\right) / \eta^{b}\right)$ and $\mu^{c}=r \mu^{b}$, denoted as $\left(\mu^{b *}, \mu^{c *}\right)$, for the single-mode strategy. Further denote the intersection between the line $\mu^{c}=r \mu^{b}$ and the line connecting $\left(\pi_{1}^{b}, \pi_{1}^{c}\right)$ and $\left(\pi_{2}^{b}, \pi_{2}^{c}\right)$ as $\left(\pi_{0}^{b}, \pi_{0}^{c}\right)$, where

$$
\pi_{0}^{b}=\frac{\pi_{1}^{c} \pi_{2}^{b}-\pi_{2}^{c} \pi_{1}^{b}}{r \pi_{2}^{b}-r \pi_{1}^{b}-\pi_{2}^{c}+\pi_{1}^{c}}, \pi_{0}^{c}=r \frac{\pi_{1}^{c} \pi_{2}^{b}-\pi_{2}^{c} \pi_{1}^{b}}{r \pi_{2}^{b}-r \pi_{1}^{b}-\pi_{2}^{c}+\pi_{1}^{c}}
$$

As $\mathcal{G}(\cdot)$ is convex, we have

$$
P_{\text {single }}^{*}=P\left(\mu^{b *}, \mu^{c *}\right)=\frac{h^{b} \mu^{b *}}{\eta^{b}}+\frac{h^{c} \mu^{c *}}{\eta^{c}} \leq P\left(\pi_{0}^{b}, \pi_{0}^{c}\right)=\frac{h^{b} \pi_{0}^{b}}{\eta^{b}}+\frac{h^{c} \pi_{0}^{c}}{\eta^{c}}
$$

Hence, the improvement is

$$
\Delta p=P_{\mathrm{bi}}^{*}-P_{\text {single }}^{*} \geq \frac{h^{b}\left(\pi_{2}^{b}-\pi_{1}^{b}\right) / \eta^{b}+h^{c}\left(\pi_{2}^{c}-\pi_{1}^{c}\right) / \eta^{c}}{\left(r \pi_{2}^{b}-r \pi_{1}^{b}-\pi_{2}^{c}+\pi_{1}^{c}\right) /\left(-\pi_{2}^{c}+r \pi_{2}^{b}\right)}
$$

For Case I.c, i.e. $r \leq \pi_{2}^{c} / \pi_{2}^{b}$, the objective function is optimized at $\left(\pi_{2}^{b}, r \pi_{2}^{b}\right)$ for the single-mode strategy,

$$
P_{\text {single }}^{*}=P\left(\pi_{2}^{b}, r \pi_{2}^{b}\right)=\frac{h^{b} \pi_{2}^{b}}{\eta^{b}}+\frac{h^{c} r \pi_{2}^{b}}{\eta^{c}}
$$

Hence, the improvement is

$$
\Delta p=P_{\mathrm{bi}}^{*}-P_{\text {single }}^{*}=\frac{h^{c}\left(\pi_{2}^{c}-r \pi_{2}^{b}\right)}{\eta^{c}}
$$

This completes the proof for the Case I in the case $\frac{h^{b} \eta^{c}}{h^{c} \eta^{b}}>-\frac{\pi_{1}^{c}-\pi_{2}^{c}}{\pi_{1}^{b}-\pi_{2}^{b}}$. The proof for the other cases is similar and is omitted here.

2) Readers can easily validate that the theoretical lower bound given in Table 2 is positive, except when $r$ is some threshold values for which cases the lower bound is 0 . 


\section{Performance of the bimodal perimeter control strategy with realistic demands}

The theoretical results in Section 4 demonstrate that the bimodal perimeter control strategy can substantially benefit network passenger mobility at steady-states. However, traffic demands vary with time in reality. Hence, the network cannot always be maintained at steady-states. Moreover, the empirical MFD and and the measured accumulations might not be accurate. Therefore, a simulation approach is advantageous because it can emulate realistic demand patterns and take into account system stochasticity and uncertainty. In this section, we conduct macroscopic simulations coded in Python to test the performance of the bimodal perimeter control strategy with realistic demands. We test the bimodal strategy using two different infrastructure configurations at the network perimeter, and compare the results to the case with a single-mode strategy. The infrastructure configurations tested in the simulations are described in Section 5.1] The simulation environment is described in Section 5.2. The results are presented and discussed in Section 5.3 .

\subsection{Infrastructure configurations}

Recall that all public transport lines travel on dedicated lanes on links approaching the perimeter, however, these dedicated lanes may be discontinued upstream of the intersection. This would affect how perimeter capacity is shared across the modes as considered in Eq7. Hence, in the simulations, this capacity share affects the constraints on the vehicle inflow into the network during transient periods. Therefore, while the theoretical calculation considers all infrastructure configurations, for simulation studies each infrastructure configuration needs to be analyzed on a case-by-case basis. We test three different infrastructure configurations in the simulations. They are respectively described below. The more general cases are also briefly discussed.

\section{Strategy 1: Single-mode strategy with mixed lanes}

In this case, for each intersection $m \in M$ with mixed incoming traffic, we have $\mu_{m}=\rho \mu_{m}^{b}+\mu_{m}^{c} \leq Q_{m}$. Summing over all intersections, we have $\sum_{m} \mu_{m}=\sum_{m} \rho \mu_{m}^{b}+\sum_{m} \mu_{m}^{c} \leq \sum_{m} Q_{m}$, i.e. $\mu=\rho \mu^{b}+\mu^{c} \leq Q$. During transient periods, if the calculated total inflow exceeds $Q$ in Eq77, evidently the adjusted inflow should be $\mu=Q$ to realize it to the maximal extent.

Strategy 2: Bimodal strategy with dedicated PT lanes immediately upstream of all perimeter intersections with PT lines $(M=I \cup L)$

In this case, for each intersection $m \in I \cup L$ with dedicated lanes (note that this includes intersections $m \in I$ with dedicated lanes for both modes, and intersections $m \in L$ with only car lanes), we have $\mu_{m}^{b} \leq Q_{m}^{b} / \rho$ and $\mu_{m}^{c} \leq Q_{m}^{c}$. Summing over all intersections, we have $\sum_{m \in I \cup L} \mu_{m}^{b} \leq \sum_{m \in I \cup L} Q_{m}^{b} / \rho$ and $\sum_{m \in I \cup L} \mu_{m}^{c} \leq \sum_{m \in I \cup L} Q_{m}^{c}$, i.e. $\mu^{b} \leq Q^{b} / \rho$ and $\mu^{c} \leq Q^{c}$. During transient periods, if the calculated public transport vehicle inflow exceeds $Q^{b} / \rho$ or the calculated car inflow exceeds $Q^{c}$, evidently the adjusted inflows should respectively be $\mu^{b}=Q^{b} / \rho$ and $\mu^{c}=Q^{c}$ to realize them to the maximal extent.

Strategy 3: Bimodal strategy with flexible-sharing strategies immediately upstream of all perimeter intersections with PT lines $(M=J \cup L)$

In this case, we assume pre-signals (Guler and Menendez, 2014a b; He et al. 2016) are implemented. The pre-signal terminates the dedicated PT lane immediately upstream of the intersection and stops vehicles at this location. Note that although such implementation occupies some road space immediately upstream of the main signal, this space is insignificant compared to the actual queue length upstream of the perimeter intersection when a perimeter control strategy is implemented. Since the inflows of both modes need to be controllable, a pre-signal for each mode is needed. The pre-signal on the PT lane controls the public transport vehicle inflow, while the pre-signal on the car lane controls the car inflow. The idea is for cars to use all the lanes at the main signal to discharge when no bus is present, so that the capacity of the intersection is 
shared in a flexible manner. In particular, the capacity at the main signal will not be wasted when the inflow of public transport vehicles is very small while a high inflow of cars is necessary for the optimal network operation. Examples of signal timing settings to optimally use the capacity of the intersection can be found in Guler and Menendez (2014a b); He et al. (2016); Yang et al. (2016); Li et al. (2013). In these papers the traffic situation of the roads upstream of the pre-signals (as compared to the dedicated lane case) are studied in detail where the speeds, travel time, and infrastructure occupancy are analyzed and compared in simulation studies both at steady states and with stochastic demands.

In reality, in most cases only the car inflow will be regulated by the pre-signal, and the pre-signal on the PT lane will be constantly green. However, the pre-signal on the PT lane remains necessary for extreme cases, such as when the network is in a gridlock state and neither additional cars nor PT vehicles should be allowed into the network. Although such situations rarely happen, the necessary infrastructure makes it feasible to manage and control traffic more effectively in such extreme cases.

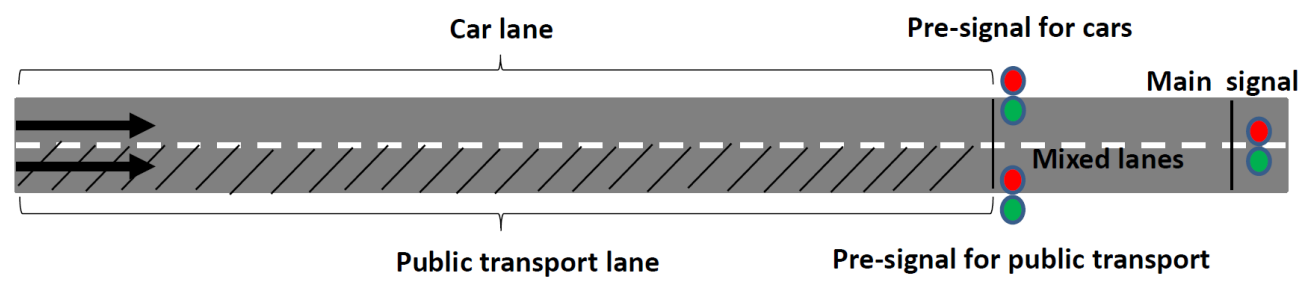

Figure 4: Configuration of pre-signals.

In general, the upper bound for car inflow at an intersection with a flexible-sharing strategy is $Q_{j}^{c}=Q_{j}-\beta_{j} \mu_{j}^{b}$ , where $\beta_{j}$ is a linear reduction factor. This linearity is justified analytically, empirically, and with simulations in previous research (Guler and Cassidy, 2012, Guler and Menendez, 2014a; He et al., 2018) on flexible-sharing strategies. Note that $\beta_{j}=\rho$ would represent a perfect sharing-system. When various degrees of PT priority are provided at the intersection level by moving public transport vehicles in front of the car queues, we have $\beta_{j}>\rho$ in general.

For each intersection $j \in J$ with flexible-sharing strategies, we have $\beta_{j} \mu_{j}^{b}+\mu_{j}^{c} \leq Q_{j}$. Summing over all intersections, we have $\sum_{j}\left(\min _{j} \beta_{j}\right) \mu_{j}^{b}+\sum_{j} \mu_{j}^{c} \leq \sum_{j} \beta_{j} \mu_{j}^{b}+\sum_{j} \mu_{j}^{c} \leq \sum_{j} Q_{j}$. Adding the car inflows from intersection $l \in L$, we have $\sum_{j}\left(\min _{j} \beta_{j}\right) \mu_{j}^{b}+\sum_{m \in J \cup L} \mu_{m}^{c} \leq \sum_{m \in J \cup L} Q_{j}$. Since $M=J \cup L$, this is equivalent to $\left(\min _{j} \beta_{j}\right) \mu^{b}+\mu^{c} \leq Q$.

During transient periods, if $\left(\min _{j} \beta_{j}\right) \mu^{b}+\mu^{c}$ exceeds $Q$, we scale $\mu^{b}$ and $\mu^{c}$ with the same factor such that $\left(\min _{j} \beta_{j}\right) \mu^{b}+\mu^{c}=Q$, so that the maximum capacity is utilized while the optimal ratio between public transport vehicle inflow and car inflow is maintained. Other possibilities exist for this case, for example giving full priority to public transport by satisfying the calculated public transport vehicle inflow, and giving the remaining capacity to cars. The exact solution can be determined empirically based on the characteristic of the network and the goal of the control. However, since most of the time the network is maintained around the steady-state and Eq 6 is satisfied, the difference between different treatments should be insignificant.

The more general cases: Bimodal strategy with both dedicated lanes and flexible-sharing strategies immediately upstream of the perimeter intersections with PT lines $(M=I \cup J \cup L)$

Lastly, we briefly discuss the more general cases when a bimodal perimeter control strategy is implemented with dedicated PT lanes upstream of some perimeter intersections and flexible-sharing strategies upstream of the others. In this case, we can similarly derive $\sum_{i} \mu_{i}^{c}+\sum_{l} \mu_{l}^{c} \leq Q^{c}, \sum_{i} \rho \mu_{i}^{b} \leq Q^{b}$, and $\sum_{j}\left(\min _{j} \beta_{j}\right) \mu_{j}^{b}+\sum_{j} \mu_{j}^{c} \leq$ 
$Q^{f}$. Summing both sides of all three constraints, a necessary condition is $\mu^{c}+\sum_{i} \rho \mu_{i}^{b}+\sum_{j}\left(\min _{j} \beta_{j}\right) \mu_{j}^{b} \leq Q$. We see this lies in between the requirement imposed by the two extreme cases, i.e. with only dedicated lanes or only flexible-sharing strategies for the pubic transport lines in the perimeter. Therefore, we expect all results to be in between these two cases. In other words, if there are more perimeter intersection with dedicated PT lanes, the results will be similar to the case where only dedicated PT lanes are implemented, and vice versa. Hence, we only test and analyze the two extreme cases.

\subsection{Simulation environment}

The city center of Zurich is chosen as the example for the simulation, i.e. all the network parameters are set to be close to the empirical values for this area. We simulate this network because a well-defined empirical 3D-MFD has been recently reported (Loder et al. 2017) for this area, hence it can be used for the simulation study to generate realistic results and make meaningful comparisons between strategies. To the authors' best knowledge, this is the first work to test a perimeter control strategy with an empirical 3D-MFD. This empirical 3D-MFD is given by Eq,2 and depicted in Fig. 1 .

This area is relatively small (around $2.5 \mathrm{~km}^{2}$ ). It has a homogeneous topology and similar hierarchy in the road network, e.g. no mixing with freeways. Moreover, public transport bunching is extremely rare in Zurich through its consistent PT priority policy. Therefore, PT vehicles are generally uniformly distributed. All these factors ensure homogeneity (both for cars and PT) in the controlled area, hence perimeter control strategies can then be applied on this area without further partitioning. Also, in the city center of Zurich, 75\% of all PT lane-km are dedicated lanes. In particular, all PT lines access the city center through dedicated lanes, hence the proposed bimodal strategy can be easily implemented with the existing infrastructure. Given the network configuration, we assume $Q^{b}=6000 \mathrm{PCE} / \mathrm{h}$ and $Q^{c}=14000 \mathrm{PCE} / \mathrm{h}$ (and total capacity $Q=20000 \mathrm{PCE} / \mathrm{h}$ ) for the network perimeter. This can represent the case where about $60 \%$ of the perimeter intersections have incoming PT lines. Cases with different network characteristics will be discussed in Section 6.2. We use a typical passenger car equivalence (PCE) factor $\rho=3$. For strategy 3, we assume that presignals of the type depicted in Figure 4 are implemented with $\beta_{j}=\rho, \forall j \in J$. The value of $\beta$ is found to have little effect on the results.

We simulate realistic traffic scenarios during a morning period of 1.5 hours. In the first half hour, the incoming car demand is relatively low. Then, in the next half hour, the incoming car demand is at peak. After that, the incoming car demands return to the initial off-peak value. Preliminary simulation tests show that a perimeter control strategy of any kind only brings significant benefit to the system when the incoming car demand is higher than 15000veh/h. Below this value, there is no need for perimeter control strategies because the network will not be congested. On the other hand, when the incoming car demand exceeds $20000 \mathrm{veh} / \mathrm{h}$, a long queue will quickly accumulate in the network perimeter. Such a long queue can cause spillbacks outside the network, and may eventually damage the operation of the entire system. Therefore, the range of peak period incoming car demands tested is between $15000 \mathrm{veh} / \mathrm{h}$ and $20000 \mathrm{veh} / \mathrm{h}$. We present results from the two extreme cases. Other cases in between also have results in between these two extreme cases. Simulation profiles of the incoming car demands $\left(d_{\text {ext }}^{c}\right)$ are shown in Figure 5 a.

The demand originating from within the network $\left(d_{\text {int }}^{c}\right)$ is assumed to be constant at $3500 \mathrm{veh} / \mathrm{h}$. Since no PT line originates from within the city center, the corresponding PT demand $\left(d_{\text {int }}^{b}\right)$ is assumed to be 0 . The initial traffic accumulation within the network at the onset of the simulation is $10 \mathrm{PT}$ vehicles and 500 cars (i.e. $\boldsymbol{n}(0)=(10,500)^{T}$ ), which is in the uncongested regime, and there is no outside accumulation for both modes (i.e. $\left.\boldsymbol{n}_{\text {out }}(0)=(0,0)^{T}\right)$.

Empirical data shows that the range of incoming PT demand for the simulated area is between 100veh/h and 200veh/h. The PT headway has little variation during the morning period. Hence, we test three levels of constant PT demands at 100veh/h, 150veh/h, and 200veh/h. The PT passenger occupancy is obtained from the Zurich public transport authority (VBZ). Statistics show that the occupancy is around 30pax/veh 
during off-peak periods and 50pax/veh during peak periods. The car passenger occupancy is obtained from the Swiss Federal Statistical Office (BFS) and assumed to be constant at 1.2pax/veh. Cases with different PT passenger occupancy will be discussed in Section 6.1. Simulation profiles of the incoming PT demands $\left(d_{\text {ext }}^{b}\right)$ and PT passenger occupancy $\left(h^{b}\right)$ are shown in Figure 5b and c, respectively.

Each strategy is tested in the simulation for all 6 incoming demand profile combinations in Figure 5 . Note that simulations after the peak period are run until all trips generated in the 1.5 hours are completed. Evidently, when the demand is higher, the simulation will be running for a longer time, because more trips are generated.
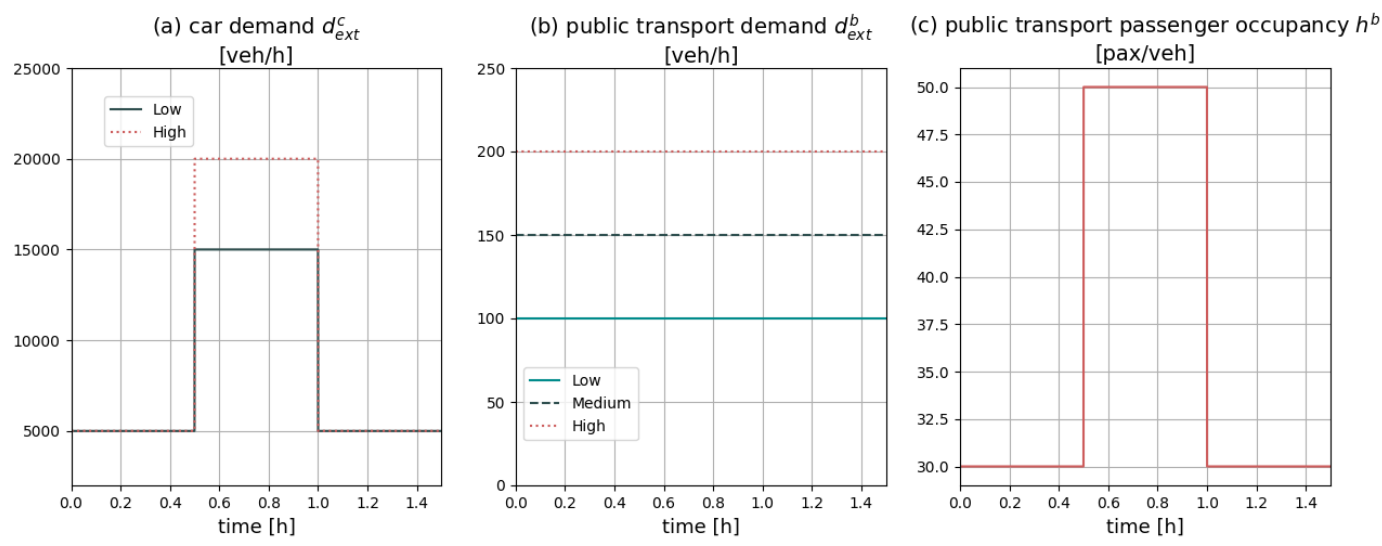

Figure 5: a. Incoming car demands $d_{\text {ext }}^{c}$. b. Incoming PT demands $d_{\text {ext }}^{b}$. c. PT passenger occupancy $h^{b}$.

For all scenarios, the network will become congested without perimeter control. In some cases, a gridlock will form. Therefore, implementing perimeter control strategies with all three strategies will benefit both modes for all these scenarios. Our goal is to compare the total system performance and mode-specific performance across the three strategies. The total system performance consists of two parts, namely the total travel time inside the network and the total waiting time outside the network perimeter (i.e. queuing due to perimeter control). The calculation of both parts are based on the assumption of a FIFO queuing system. Moreover, to make results comparable across different demands, all the following results are only based on the trips generated during the first 1.5 hours of simulation period, irrespective of the simulation running time, i.e. trips generated after the first 1.5 hours are not taken into account for the calculations.

To test the strategy performance in simulations, a specific controller needs to be applied. Many controllers have been developed for perimeter control, for example Proportional-Integral-Derivative (PID) controllers (Keyvan-Ekbatani et al. 2012) and Model predictive control (MPC) controllers (Geroliminis et al., 2013 Yang et al. [2018b). Note that it is not the purpose of this paper to design an optimal controller for the bimodal strategy. Instead, this paper shows a proof-of-concept to illustrate the general properties of the proposed strategy, and compare it with the single-mode strategy to demonstrate its advantage. This is crucial as a first step, because implementation of the proposed strategy does not only involve adjustment of the controllers (i.e. signal timings), but can also involve some infrastructure alterations. When the proposed bimodal strategy is considered beneficial by the policy makers, similar controller design techniques as those in the above literature can then be adopted to optimize the controller.

To this end, we compare the performance of a single-mode control strategy with that of a bimodal control strategy, applying the same type of controller. We implement a PID controller Åström and Hägglund, 2006), which is a feedback control mechanism applied extensively in both the industry and the research community (Shabani et al. 2013, Yu and Rosen, 2013). A PID controller aims to reduce the difference between the desired states and the measured states for some system variables, based on the proportional, integral, and derivative terms. It is shown in McMillan and Cameron (2003) that the PID can outperform 
MPC for unmeasured disturbances, which are often associated with perimeter control. Moreover, note that a controller with many parameters is undesirable. If the single-mode and bimodal strategies are both implemented with such complicated controllers, the differences in control performance could then arguably be due to parameter settings of the controllers, rather than the strategy design. This hinders the comparison of the general characteristics between the two strategies, which should be independent of the specific controllers applied. Therefore, a full PID controller is not necessary. Instead, the proportional controller, a simple yet effective type of the PID controller, is used in this paper. The proportional controller has been widely applied in the industry, e.g. in transportation (Zheng et al., 2012) and robotics (Amsuess et al. 2015). More details about the proportional controller used in our simulations can be found in Appendix A

\subsection{Results and analysis}

The system performance of the three strategies is summarized in Table 4 for comparison. The system performance includes average travel time inside the network, average waiting time outside the network, and the sum of the two, i.e. the total cost. To better understand how the strategies affect the system performance over the simulated period, relevant network states for the case with high incoming car demand (20000veh/h) and low incoming PT demand $(100 \mathrm{veh} / \mathrm{h})$ are plotted in Figure 6 as an illustrative example.

Table 4: Comparison of the system performance (travel time inside the network; waiting time outside the network; total cost) of the three perimeter control strategies (PS: Pre-signal; DL: Dedicated lanes; ML: Mixed-lanes) with six demand profiles. All results are in seconds. Best strategy for each performance metrics is in bold. Best strategy for least average passenger total cost is shaded.

\begin{tabular}{|c|c|c|c|c|c|c|c|c|c|c|c|}
\hline \multicolumn{2}{|c|}{ demand } & \multicolumn{3}{|c|}{ cars } & \multicolumn{3}{|c|}{$\mathrm{PT}$} & \multicolumn{4}{|c|}{ passenger } \\
\hline car & $\mathrm{PT}$ & & ML & $\mathrm{DL}$ & PS & ML & DL & PS & ML & $\mathrm{DL}$ & $\mathrm{PS}$ \\
\hline \multirow{9}{*}{ low } & \multirow{3}{*}{ low } & inside & 338 & 339 & 339 & 406 & 407 & 407 & 364 & 354 & 354 \\
\hline & & outside & 78 & 106 & 97 & 56 & 3 & 3 & 75 & 78 & 71 \\
\hline & & total & 417 & 445 & 436 & 462 & 410 & 410 & 439 & 432 & 426 \\
\hline & \multirow{3}{*}{ mid } & inside & 361 & 366 & 367 & 411 & 412 & 412 & 397 & 381 & 381 \\
\hline & & outside & 153 & 196 & 187 & 120 & 7 & 7 & 146 & 129 & 122 \\
\hline & & total & 514 & 563 & 554 & 531 & 419 & 419 & 544 & 509 & 503 \\
\hline & \multirow{3}{*}{ high } & inside & 380 & 393 & 394 & 410 & 418 & 419 & 423 & 403 & 404 \\
\hline & & outside & 252 & 322 & 313 & 226 & 31 & 31 & 246 & 196 & 191 \\
\hline & & total & 632 & 715 & 707 & 635 & 449 & 450 & 669 & 600 & 596 \\
\hline \multirow{9}{*}{ high } & \multirow{3}{*}{ low } & inside & 356 & 360 & 362 & 400 & 412 & 412 & 385 & 371 & 372 \\
\hline & & outside & 374 & 478 & 452 & 320 & 9 & 9 & 366 & 368 & 348 \\
\hline & & total & 730 & 838 & 815 & 721 & 421 & 421 & 752 & 738 & 720 \\
\hline & \multirow{3}{*}{ mid } & inside & 357 & 379 & 380 & 403 & 415 & 415 & 406 & 389 & 390 \\
\hline & & outside & 510 & 641 & 627 & 466 & 10 & 9 & 501 & 442 & 432 \\
\hline & & total & 867 & 1020 & 1007 & 869 & 424 & 425 & 907 & 831 & 822 \\
\hline & \multirow{3}{*}{ high } & inside & 352 & 404 & 405 & 406 & 418 & 419 & 424 & 410 & 411 \\
\hline & & outside & 664 & 829 & 820 & 646 & 31 & 31 & 660 & 524 & 518 \\
\hline & & total & 1016 & 1233 & 1224 & 1052 & 449 & 450 & 1084 & 934 & 929 \\
\hline
\end{tabular}

Noticeably, the differences in results across different strategies and different demands are much larger for the average waiting time outside the network than for the average travel time inside the network. All three strategies are able to maintain the network accumulation around the optimal level at all tested demands. Evidently, when the demand is higher, the average travel times inside the network are a little longer, but the network is still in the non-congested regime and operates around the optimum car accumulations. In contrast, if no perimeter control strategy is applied, simulation tests confirm that the network will be congested during the peak period, resulting in much higher travel time within the network for both modes. This confirms conclusions from previous studies (Ampountolas et al., 2017) that applying perimeter control strategies improves the traffic operation for both modes within the network. This is not surprising. We will 

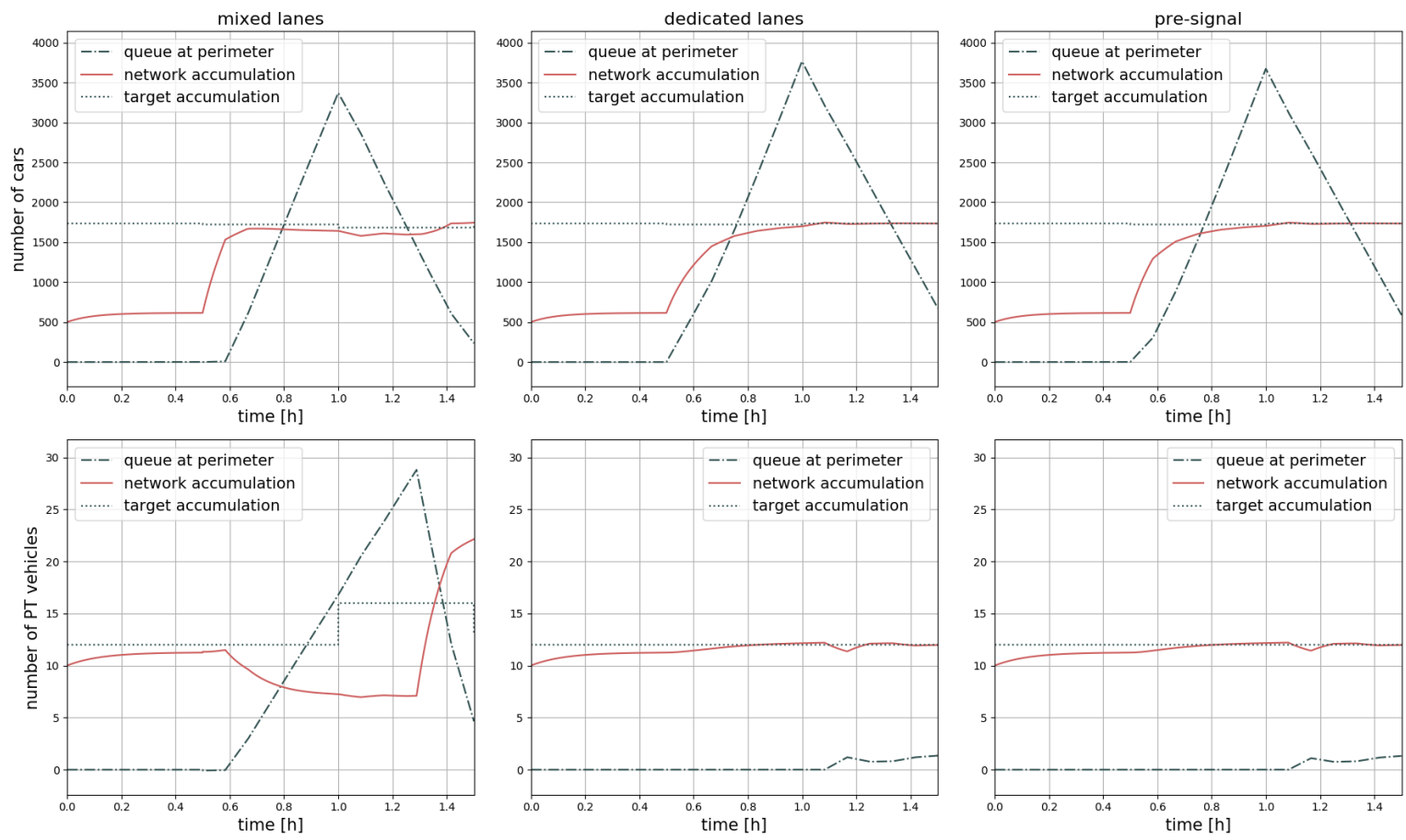

Figure 6: Comparison of the three perimeter strategies in a realistic morning peak period for the case with high incoming car demand 20000veh/h and low incoming PT demand 100veh/h.

focus our analysis on comparing the three strategies. Note that, while the travel times inside the network have very small differences, the average waiting time outside the network varies significantly across different strategies and different demands. A detailed analysis is provided below.

Looking at the average car waiting time outside, with all three strategies the waiting times increase significantly with increasing demands. This is true in general for any perimeter control strategy, because at higher demands, more vehicles needs to be held up in the perimeter for the network to be operating at optimal without getting seriously congested. Not surprisingly, mixed lanes always result in the smallest car waiting time among the three strategies. When the PT frequency is low, pre-signals have similar average car waiting times as mixed lanes, which is smaller than dedicated lanes. This is due to the capacity sharing property of pre-signals as a flexible-sharing strategy to maximally utilize the capacity of the perimeter intersections.

Looking at the average PT waiting time outside, not surprisingly, mixed lanes always result in the largest PT waiting time. In fact, the waiting time increases significantly with increasing demands, because PT vehicles receive no priority and will be queued outside the perimeter with the cars. This can be observed from Figure 6 for mixed lanes. Starting from $t=0.5 \mathrm{~h}$, a significant number of PT vehicles will be queued in the perimeter. This also hinders the network from reaching its target PT accumulation, since there is no possibility to let in more PT vehicles once the target car accumulation is reached. In contrast, pre-signals and dedicated lanes result in much smaller PT waiting times and a small PT accumulation outside the network. Note that the PT operations with these two strategies are exactly the same. Therefore, PT priority is provided with both bimodal strategies, and a similar level of priority is achieved. Moreover, since PT inflow can be independently regulated with bimodal perimeter control, the network can always operate around the target PT accumulation. Therefore, overall we conclude that a bimodal strategy is more efficient in achieving the traffic management and control goals than a single-mode approach.

Looking at average passenger waiting time outside, both bimodal strategies outperform the single-mode 
strategy with mixed lanes for all tested demands. This is due to the space efficiency of the PT mode and the PT priority provided by the bimodal strategies. Moreover, implementing a bimodal strategy with pre-signals always outperform dedicated lanes. This is because pre-signals can provide a similar level of PT priority as dedicated lanes, while achieving a higher perimeter car inflow.

Since the variations are much larger for the average waiting time outside the network than for the average travel time inside the network, the total travel cost is dominated by the waiting time outside. Therefore, not surprisingly the bimodal strategies (i.e. strategy 2 and 3) outperform the single-mode strategy (i.e. strategy 1) from a passenger perspective for all tested demands. Moreover, the bimodal strategy can provide significant PT priority, especially when the car demand is high, as the total travel cost is much smaller with PT than with cars, when either bimodal strategy is implemented. Lastly, comparing the two bimodal strategies, we conclude that implementing pre-signals (i.e. strategy 3) outperform dedicated lanes from a passenger perspective for all tested demands. Further simulation tests show that all qualitative results (i.e. comparison between three strategies) remains valid when there is stochasticity and uncertainty in the system. More details can be found in Appendix B.

\section{Sensitivity to different traffic scenarios}

The simulation results in Section 5 demonstrates that from a passenger mobility perspective:

- The bimodal perimeter control strategy always outperforms the single-mode strategy, i.e. both Strategy 2 and Strategy 3 outperform Strategy 1. This further confirms our theoretical results in Section 4

- Implementing the bimodal strategy with pre-signals always outperforms dedicated lanes, i.e. Strategy 3 outperforms Strategy 2.

However, different transportation networks have different traffic scenarios, which might differ from that of Zurich city center. In order to offer general recommendations to the policy makers, we need to evaluate the validity of the above conclusions for a range of different traffic scenarios. Moreover, implementing the bimodal strategy might require additional infrastructure investment, for example some lanes in the perimeter need to be converted to dedicated lanes. When flexible-sharing strategies such as pre-signals are considered, the infrastructure investment and control complexities are even higher. Therefore, even if a bimodal strategy is advantageous, it is necessary to quantify the benefit for different traffic scenarios, so that policy makers can decide whether the investment is justified. To this end, a sensitivity analysis is carried out in this section to answer the following two questions for a range of traffic scenarios:

- If we are considering implementing perimeter control, how much extra benefit does a bimodal perimeter control strategy offer, compared to a single-mode one?

- If we are considering implementing a bimodal perimeter control strategy, how much extra benefit does the additional investment to implement flexible-sharing strategies offer, compared to implementing only dedicated lanes?

The difference between the average passenger travel cost (travel time inside the network plus the waiting time outside the network) is calculated for different strategies with different scenarios to answer these two questions. The difference between mixed lanes (Strategy 1) and dedicated lanes (Strategy 2) is calculated to answer the first question. The difference between dedicated lanes (Strategy 2) and flexible-sharing strategies (Strategy 3) is calculated to answer the second question. Note that in both cases, when the difference is smaller than 0 , the former strategy performs better than the latter one. 
We use a one-at-a-time (OAT) method with the simulation model developed in Section 5 for the sensitivity analysis of multiple parameters, i.e. when the sensitivity of each parameter is tested, all other parameters are held constant. Other more complex methods (e.g. Ge and Menendez (2014, 2017); Ge et al. (2015)) can be used in the future to account for the combined effects of multiple parameters. Results with PT passenger occupancy and proportion of perimeter intersections with PT lines are presented in this section, respectively. Other parameters, such as the flexible-sharing strategy reduction factor and duration of the peak period, are found to have little effect on the qualitative conclusions, i.e. they do not affect the comparison between strategies.

\subsection{PT passenger occupancy}

Since one primary purpose for implementing a bimodal perimeter control strategy is to provide PT priority, the PT passenger occupancy $\left(h^{b}\right)$ will be a critical parameter in deciding how much priority should be given to PT. Intuitively, we expect the bimodal strategy to become more beneficial as the PT passenger occupancy gets higher. To quantify this, we analyze peak period PT passenger occupancy ranging from 30pax/veh to 70pax/veh. The results are summarized in Figure 7.
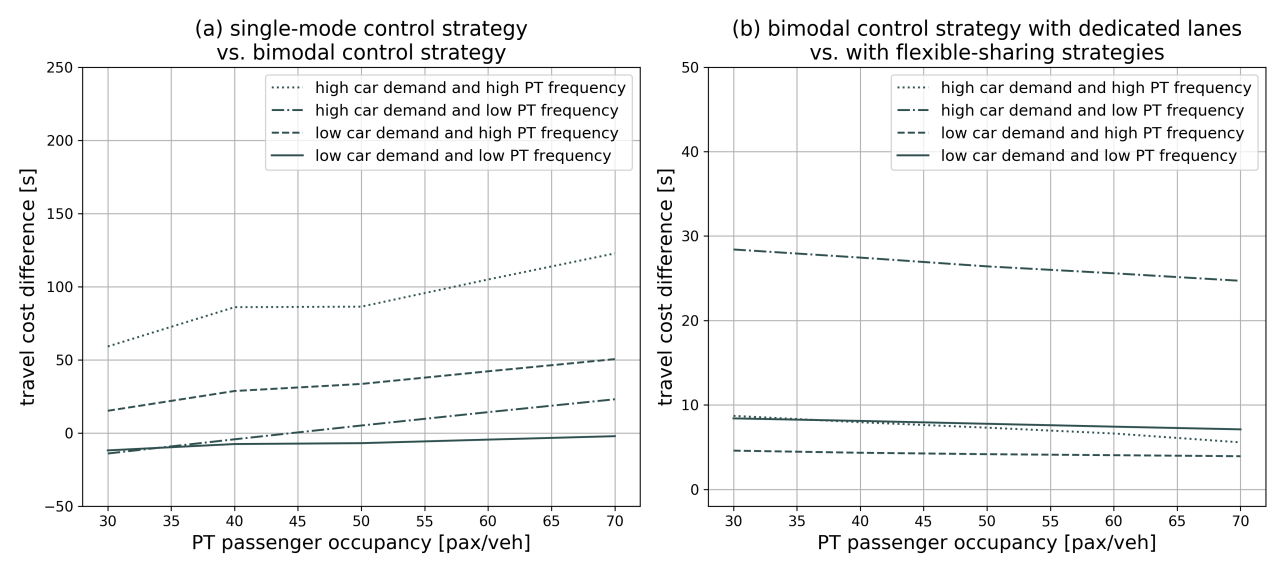

Figure 7: Sensitivity to PT passenger occupancy: a. Difference between the average passenger total travel cost with mixed lanes and dedicated lanes at different PT passenger occupancies; b. Difference between the average passenger total travel cost with dedicated lanes and flexible-sharing strategies at different PT passenger occupancies.

From Figure 7a we see that for all tested scenarios, the increase in system benefit from implementing the bimodal strategy with dedicated lanes is approximately linear with the increase in PT passenger occupancy. For cases with a high PT frequency, the bimodal strategy is always beneficial, even at relatively low PT occupancy levels. However, for cases with a low PT frequency, the bimodal strategy can harm the system, when the PT occupancy is very low. This is not surprising. When PT frequency and passenger occupancy are both low, PT priority and space allocation are not justified. Comparing the two bimodal strategies in Figure 7b, implementing flexible-sharing strategies always offers extra benefit. Notice that flexible-sharing strategies offer the most extra benefit in the case with high car demand and low PT frequency. This additional benefit more than compensates the harm from implementing dedicated lanes at low PT passenger occupancy levels. Hence, in these cases, the bimodal strategy with flexible-sharing strategies performs better than a single-mode one.

In summary, it is always more beneficial to implement a bimodal perimeter control strategy compared to a single-mode one, irrespective of the PT passenger occupancy. However, if the PT passenger occupancy and demand are both low, it is necessary to implement flexible-sharing strategies for the bimodal strategy. When both dedicated lanes and flexible sharing strategies are applicable, implementing flexible-sharing strategies offers an approximately constant amount of additional benefit irrespective of the PT passenger occupancy. 


\subsection{Proportion of perimeter intersections with PT lines}

Recall that a necessary infrastructure requirement when implementing a bimodal perimeter control strategy is that all PT lines need to enter the controlled area through dedicated lanes. Therefore, if originally all perimeter intersections have mixed lanes to access the controlled area, the number of lanes which need to be converted to dedicated PT lanes is higher when the proportion of perimeter intersections with PT lines is higher. This is associated with proportionally higher infrastructure investment, hence it is an important parameter to analyze. Furthermore, this determines the perimeter capacity share between modes (i.e. $Q^{c}$ and $Q^{b}$ for a given $Q$ ) when dedicated lanes are implemented. This capacity share then determines the constraints on the inflows. The calculated optimal inflow might not be realized if the perimeter capacity of the corresponding mode is too low, resulting in less than optimal network accumulation and unnecessary queues outside the perimeter.

We analyze the proportion of perimeter intersections with PT lines range from $20 \%$ (i.e. a network where only a few perimeter intersections have PT lines) to $100 \%$ (i.e. a network where all perimeter intersections have PT lines). When dedicated lanes are implemented on these intersections for the bimodal strategy, the perimeter PT capacity correspondingly ranges from 2000PCE/h to 10000PCE/h, assuming the two modes equally split the capacity at the shared intersections. Therefore, the potential network PT capacity share ranges from 0.1 to 0.5 . The results are summarized in Figure 8. Note that $Q^{b} / Q=0$ (i.e. $Q^{c}=20000 \mathrm{PCE} / \mathrm{h}$ ) corresponds to the case when there is no PT lines going through the network perimeter, hence is irrelevant to the topic of this paper.

From Figure $8 \mathrm{a}$ we see that implementing the bimodal strategy with dedicated lanes is beneficial when not many perimeter intersections have PT lines. In this case, only a small number of dedicated lanes need to be implemented. This means that a relatively small investment could result in significant benefit for the network. Also, when the PT frequency is high, it is always beneficial to implement dedicated lanes for all tested scenarios. This is not surprising because the PT priority provided will increase the road space efficiency. On the other hand, when the PT frequency is low and many perimeter intersections have PT lines, implementing the bimodal strategy with dedicated lanes can harm the system. This is because the network perimeter will be dedicating too much capacity to PT, while leaving inadequate capacity for cars.

From Figure 8 $\mathrm{p}$ we see that for all tested scenarios it never harms the system to implement flexible-sharing strategies on the dedicated lanes. When many perimeter intersections have PT lines, the benefit to implement flexible-sharing strategies on all of them is significant. This is because flexible-sharing strategies can significantly increase the car inflow compared to dedicated lanes, while still providing a similar level of PT priority. Admittedly, more flexible-sharing strategies need to be implemented when there are more dedicated lanes, which requires a larger investment. However, while the amount of investment required usually increases at a decreasing rate due to economies of scale, the increase in benefit offered by flexible-sharing strategies is clearly increasing at an increasing rate. This is especially the case when the PT frequency is low. Recall that with low PT frequencies, implementing dedicated lanes may harm the system. However, comparing Figure 8 $\mathrm{a}$ and Figure $8 \mathrm{~b}$, the benefit from additionally implementing flexible-sharing strategies on these intersections more than compensates the harm from implementing dedicated lanes on them. Hence, in these cases, the bimodal strategy with flexible-sharing strategies still performs better than a single-mode one.

In summary, it is always more beneficial to implement a bimodal perimeter control strategy compared to a single-mode one, irrespective of the proportion of perimeter intersections with PT lines. However, if many perimeter intersections have PT lines, it is necessary to implement flexible-sharing strategies for the bimodal strategy. When both dedicated lanes and flexible sharing strategies are applicable, implementing the bimodal strategy with these two infrastructure configurations results in similar system performance. 

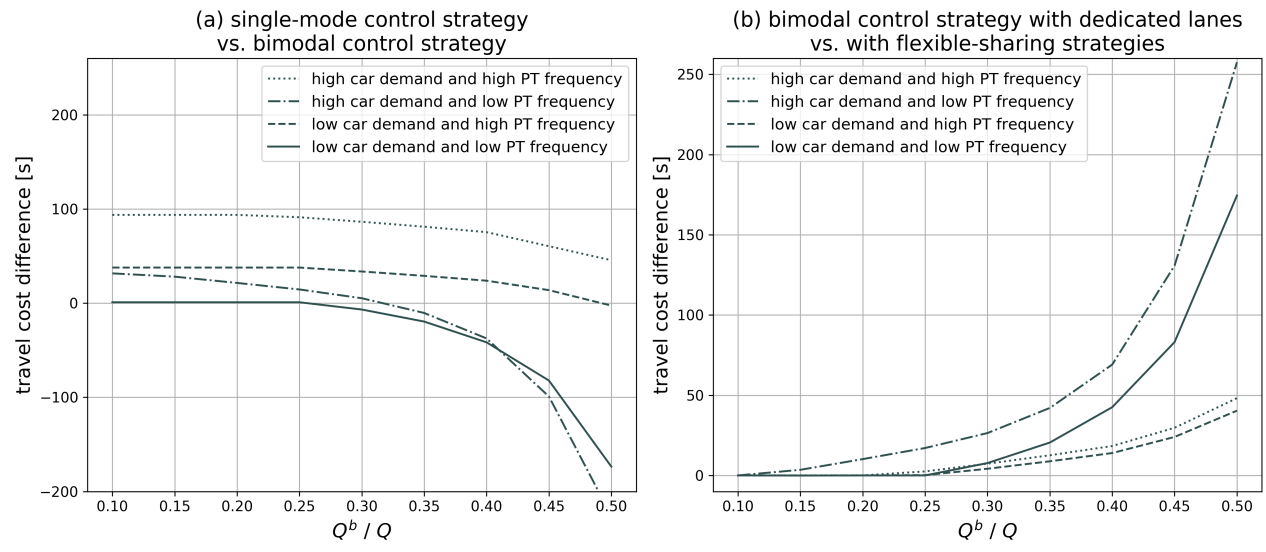

Figure 8: Sensitivity to the proportion of perimeter intersections with PT lines (i.e. perimeter PT capacity share after dedicated lanes are implemented for these PT lines): a. Difference between the average passenger total travel cost with mixed lanes and dedicated lanes at different potential perimeter PT capacity shares; b. Difference between the average passenger total travel cost with dedicated lanes and flexible-sharing strategies at different potential perimeter PT capacity shares.

\section{Conclusions and discussions}

The contribution of this paper is twofold. First, methodologically, we develop an integrated and systematic framework for the optimization of bimodal urban networks using 3D-MFDs. Such a methodological framework is necessary due to the complexities of bimodality, i.e. the two modes are often coupled. This framework takes into consideration multiple goals of decision makers, which are often conflicting. We propose to first obtain the pareto frontier for conflicting objectives using 3D-MFDs, and then evaluate the optimal solution for a linear objective such as the passenger flow. In this way, effective strategies can be designed for the planning, management, and control of bimodal networks. In particular, strategies to provide public transport priority on the network level can be holistically evaluated.

Second, we propose, model, and analyze one such strategy, a bimodal perimeter control strategy, as an application of the methodological framework. The proposed strategy addresses a pressing problem of the existing perimeter control schemes: public transport vehicles will be queuing with the cars in the perimeter and hence blocked from entering the network. This impairs the service quality of public transport. To the authors' best knowledge, the proposed bimodal strategy is the first strategy in literature which enables public transport vehicles to bypass the car queues in the perimeter of the network. Adopting this strategy, the inflows of public transport and cars can be controlled independently (i.e. both inflows are controllable), the network traffic can be managed more efficiently, and public transport priority can be provided in the perimeter.

To analytically evaluate the performance of the bimodal strategy, the interactions between the two modes, both within the network and in the network perimeter, are mathematically modelled. Within the network, we develop a bimodal perimeter control model using the 3D-MFD to quantify the complex dynamics of the two modes. In the network perimeter, we model how the perimeter infrastructure imposes constraints to the traffic inflow with different degrees of coupling between the two modes. Overall, the mathematical model enables the quantitative analysis of a general bimodal perimeter control strategy.

The performance of the bimodal strategy is evaluated both analytically and with simulations. Analytically, we apply the proposed methodological framework to evaluate the performance of the strategy at steadystates. We compare its performance to the case when a single-mode strategy is implemented, and derive the theoretical lower bound for improvement in passenger flow. Furthermore, we use a macroscopic simulation based on the city center of Zurich, with its empirical 3D-MFD, to evaluate the bimodal strategy with realistic 
demands. This is also the first test of perimeter control using an empirical 3D-MFD.

We have chosen a macroscopic approach using 3D-MFD for modeling and analysis because it offers four main benefits. First, the 3D-MFD offers a macroscopic view of the bi-modal system without the need for tracking individual vehicles. Although a microscopic approach can also be used, it would introduce too many factors that add unnecessary complexity to the analysis. Second, a 3D-MFD enables us to develop an integrated and systematic framework in Section 2 for the optimization of bimodal urban networks. Third, a 3D-MFD models the interaction between public transport vehicles and cars. Therefore, with a 3D-MFD, we can model how the traffic system evolves with the proposed bimodal strategies. This helps us to model the bimodal perimeter control strategy in Section 3. Fourth, the 3D-MFD provides an analytical tool to evaluate and optimize the performance of bimodal perimeter control strategies. This helps us in Section 4 to derive the theoretical lower bound for improvement when a bimodal perimeter control strategy is implemented, and also to calculate the optimal network traffic accumulations, which are used in the controllers in Section 5.

Results from both the analytical and simulation studies show that the bimodal strategy always performs better than the single-mode strategy in terms of passenger mobility. Most importantly, the bimodal strategy differentiates the public transport mode and the car mode, with much smaller queueing time outside the network for public transport. To offer recommendations to policy makers on whether to implement the bimodal strategy with dedicated lanes or with flexible-sharing strategies, we compared their performance in a variety of traffic scenarios. Results show that the extra benefit with flexible-sharing strategies is most significant when the public transport passenger occupancy and demand are both low, or if many perimeter intersections have public transport lines going into the network. In other cases, dedicated lanes can be an efficient solution, as implementing flexible-sharing strategies comes with additional infrastructure investment without significant extra benefit.

It should be noted that both the methodological framework and the bimodal strategy model are independent of the shape of the 3D-MFD, the controller, and the traffic management and control goals. Hence, we expect them to be generally applicable and the qualitative conclusions generally valid. Overall, implementing the bimodal strategy not only improves the bimodal traffic within the network, but also prioritizes public transport. It is a promising traffic management and control strategy which can improve the overall urban mobility. In the long run, good public transport service can encourage more people to commute with public transport into the city, which can further reduce the congestion both within and outside the network, and eventually shift the transportation system to a more sustainable state.

\section{Acknowledgement}

This research has been partially funded by the Swiss National Science Foundation (SNSF) under the project name SignBus, contract 205121_150180.

\section{Appendix A. The proportional controller in simulations}

The controller can be designed according to the target accumulation $\tilde{\boldsymbol{n}}$ calculated from Eq 5 and Eq 6 . Note that although in theory the state of the system $\boldsymbol{n}$ can be measured in real time, and the corresponding control can be calculated and varied in real time, this is not how it is implemented in practice for perimeter control. Firstly, perimeter control is usually realized with pulsed servers (e.g. traffic signals). Hence, the control $\boldsymbol{\mu}$ is only meaningful in a time-average sense. Secondly, it takes some time for the network to stabilize before the accumulation $\boldsymbol{n}$ can be correctly measured by available detectors. For these reasons, the controller is updated in real time for every control application period of duration $\omega$ (e.g. 5 minutes). Note that the control design period $(\Omega)$ contains multiple control application periods $(\omega)$ because controllers are designed based 
on predictions on larger time scales (e.g. 30 minutes). The prediction is based on real-time measurement (from connected vehicles, loop detectors, etc.) and/or historical data.

To obtain the controller, we first linearize the non-linear dynamic equations Eq 3 around the corresponding steady-state $\tilde{\boldsymbol{n}}$ using the first order Taylor expansion, then discretize the resulting linear equation with a discretization step of $\omega$, and obtain EqA.1.

$$
\Delta \boldsymbol{n}((k+1) \omega)=\boldsymbol{A} \Delta \boldsymbol{n}(k \omega)+\boldsymbol{B} \Delta \boldsymbol{\mu}(k \omega)
$$

where $k$ represents the index of the control application period. The deviation from the target accumulation at steady-state is $\Delta \boldsymbol{n}=\boldsymbol{n}-\tilde{\boldsymbol{n}} \in \mathbb{R}^{2 \times 1}$. The deviation from steady-state control is $\Delta \boldsymbol{\mu}=\mu-\tilde{\mu} \in \mathbb{R}$ for single-mode control, and $\Delta \boldsymbol{\mu}=\boldsymbol{\mu}-\tilde{\boldsymbol{\mu}} \in \mathbb{R}^{2 \times 1}$ for bimodal control. $\boldsymbol{A}$ and $\boldsymbol{B}$ are the linear matrices evaluated at $\tilde{\boldsymbol{n}}$. $\boldsymbol{A}$ is the state matrix, and $\boldsymbol{B}$ is the control matrix, where $\boldsymbol{B}=\boldsymbol{\theta} \in \mathbb{R}^{2 \times 1}$ for single-mode control, and $B \in \mathbb{R}^{2 \times 2}$ is the identity matrix for bimodal control.

The proportional controller assumes that the deviation from steady-state control $\Delta \boldsymbol{\mu}$ should be set proportionally to the deviation from the steady-state accumulation $\Delta \boldsymbol{n}$. The proportionality is quantified by the control gain factor $K$, hence,

$$
\Delta \mu=-K \Delta n
$$

where $\boldsymbol{K} \in \mathbb{R}^{1 \times 2}$ for single-mode control ; $\boldsymbol{K} \in \mathbb{R}^{2 \times 2}$ for bimodal control.

Our control objective is to reach the target accumulation $\tilde{\boldsymbol{n}}$ and control $\tilde{\boldsymbol{\mu}}$ quickly, and maintain the network at the steady-state with little fluctuations. Therefore, an appropriate objective function is the weighted squared deviations from the target on the infinite horizon:

$$
\mathcal{L}=\sum_{k=0}^{\infty}\left(\Delta \boldsymbol{n}^{T}(k \omega) \boldsymbol{S} \Delta \boldsymbol{n}(k \omega)+\Delta \boldsymbol{\mu}(k \omega)^{T} \boldsymbol{R} \Delta \boldsymbol{\mu}(k \omega)\right)
$$

where $\boldsymbol{S}$ and $\boldsymbol{R}$ are weighting factors 1 which stabilize the network accumulation $\boldsymbol{n}$ and control $\boldsymbol{\mu}$, respectively. $\boldsymbol{S}$ and $\boldsymbol{R}$ respectively represent the trade-off between the network accumulation and control, and the tradeoff between the public transport and car mode. The optimal proportional controller which minimizes the objective function $\mathcal{L}$ has gain factor $\boldsymbol{K}=\boldsymbol{R}^{-1} \boldsymbol{B}^{T} \boldsymbol{X}$, where $\boldsymbol{X} \in \mathbb{R}^{2 \times 2}$ is a positive definite matrix satisfying the algebraic Riccati equation associated with EqA.1 A.3.

$$
\boldsymbol{X}=\boldsymbol{S}+\boldsymbol{A}^{T}\left(\boldsymbol{X}-\boldsymbol{X} \boldsymbol{B}\left(\boldsymbol{R}+\boldsymbol{B}^{T} \boldsymbol{X} \boldsymbol{B}\right)^{-1} \boldsymbol{B}^{T} \boldsymbol{X}\right) \boldsymbol{A}
$$

Overall, the control process can be summarized graphically in the control diagram in Figure A.9.

All three perimeter control strategies are implemented with the proportional controllers designed according to Figure A.9. The controllers have $\boldsymbol{R}=[[0.5,0] ;[0,1]]$ for bimodal control; $\boldsymbol{R}=0.1$ for single-mode control; $\boldsymbol{S}=[[1,0] ;[0,5]]$. These parameters are calibrated by optimizing each strategy following a cross validation procedure to minimize the total travel cost both inside and outside the network. The design and application periods are chosen as $\omega=5 \mathrm{mins}$; and $\Omega=30 \mathrm{mins}$, respectively.

\footnotetext{
${ }^{1}$ Note that for single-model control, $\boldsymbol{R} \in \mathbb{R}$; for bimodal control, $\boldsymbol{R} \in \mathbb{R}^{2 \times 2} . \boldsymbol{S} \in \mathbb{R}^{2 \times 2}$ for both cases.
} 


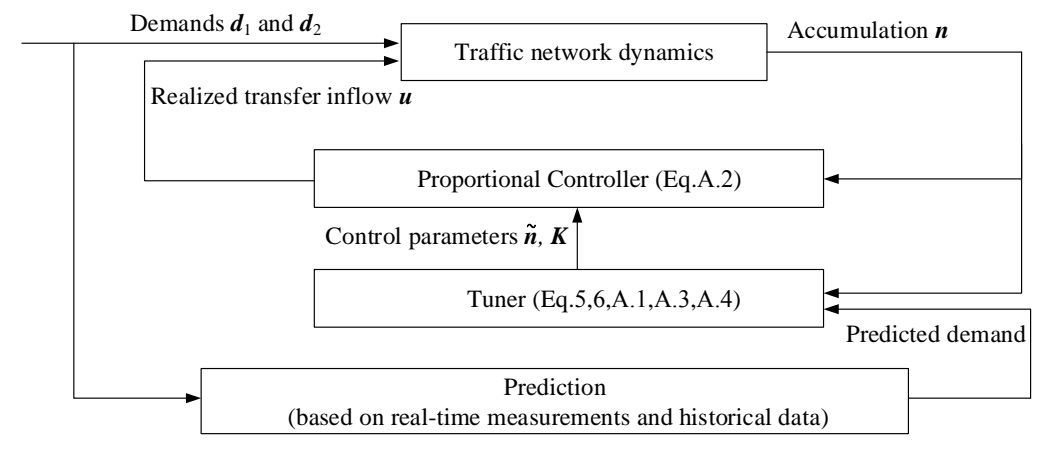

Figure A.9: Control diagram for bimodal perimeter control with a proportional controller.

\section{Appendix B. Performance with noise}

We test the performance of the three strategies with stochasticity and uncertainty in the system, i.e. in a noisy environment. This is necessary because, on one hand, the real-world traffic system is highly stochastic. The actual traffic demand fluctuates over time, and the evolution of the traffic system cannot be fully characterized with an MFD. On the other hand, due to limited coverage of traffic detectors, errors in measurement, and data corruption in transmission, we do not have perfect information on the accumulations inside the network. Therefore, it is important to evaluate the three strategies in a more realistic noisy environment before realworld deployment. We evaluate the system performance at a moderate noise level. In particular, we consider three types of noises: the stochasticity in the MFD, the variation in demand, and the error in the estimated accumulations.

For the stochasticity in the MFD, we assume the actual outflows from the network for both buses and cars follow a uniform distribution between $0.9 g(n)$ and $1.1 g(n)$. For the variation in demand, we assume it follows a Gaussian distribution with mean 0 and a standard deviation of $10 \%$ of the average demand. For the error in the estimated accumulations, we assume it follows a Gaussian distribution with a mean of 0 and a standard deviation of $10 \%$ of the actual value. These noise variables are assumed to be independent of each other. Essentially, the noises make the actual values different from the theoretical values used to devise the perimeter controller, resulting in suboptimal performance. To evaluate how these noises in the system affect system performance, all other simulation settings are the same as in Section 5.3, and results with and without noises are compared.

The system performance of the three strategies in the simulated noisy environment is summarized in Table B.5 for comparison. Table B.5 also compares all results in the noisy environment to the results without noises presented in Table 4 . To better understand the system performance over the simulated period, relevant network states over the simulated period for the case with high incoming car demand $(20000 \mathrm{veh} / \mathrm{h})$ and low incoming PT demand (100veh/h) are plotted in Figure B.10 as an illustrative example.

It can be seen that the travel costs in Table B.5 are slightly higher due to stochasticity and uncertainty in the system. Nevertheless, the conclusions in Section 5.3 still hold. Figure B.10 shows that all three strategies are still able to maintain the car accumulations around the target, and the bimodal strategies are still able to maintain the PT accumulation also around the target. Therefore, we can conclude that all the three strategies are robust to moderate noises. In realistic traffic scenarios with noises, the bimodal strategies (i.e. strategy 2 and 3) still outperform the single-mode strategy (i.e. strategy 1), and implementing pre-signals for the bimodal strategy (i.e. strategy 3 ) still outperforms dedicated lanes. 
Table B.5: Comparison of the system performance (travel time inside the network; waiting time outside the network; total cost) of the three perimeter control strategies (PS: Pre-signal; DL: Dedicated lanes; ML: Mixed-lanes) with six demand profiles in a noisy environment. All results are in seconds. Percentage increase in total cost due to noise is in parentheses. Best strategy for each performance metrics is in bold. Best strategy for least average passenger total cost is shaded.

\begin{tabular}{|c|c|c|c|c|c|c|c|c|c|c|c|}
\hline \multicolumn{3}{|c|}{ demand } & \multicolumn{3}{|c|}{ cars } & \multicolumn{3}{|c|}{$\mathrm{PT}$} & \multicolumn{3}{|c|}{ passenger } \\
\hline car & $\mathrm{PT}$ & & ML & $\mathrm{DL}$ & PS & ML & DL & PS & ML & $\mathrm{DL}$ & $\mathrm{PS}$ \\
\hline \multirow{9}{*}{ low } & \multirow{3}{*}{ low } & inside & $\begin{array}{r}\mathbf{3 3 8} \\
(0 \%)\end{array}$ & $\begin{array}{r}\mathbf{3 3 8} \\
(0 \%)\end{array}$ & $\begin{array}{r}\mathbf{3 3 8} \\
(0 \%)\end{array}$ & $\begin{array}{r}409 \\
(0 \%)\end{array}$ & $\begin{array}{r}\mathbf{4 0 8} \\
(0 \%)\end{array}$ & $\begin{array}{r}\mathbf{4 0 8} \\
(0 \%)\end{array}$ & $\begin{array}{r}365 \\
(0 \%)\end{array}$ & $\begin{array}{r}\mathbf{3 5 4} \\
(0 \%)\end{array}$ & $\begin{array}{r}\mathbf{3 5 4} \\
(0 \%)\end{array}$ \\
\hline & & outside & $\begin{array}{r}106 \\
(+36 \%) \\
\end{array}$ & $\begin{array}{r}131 \\
(+24 \%) \\
\end{array}$ & $\begin{array}{r}131 \\
(+35 \%) \\
\end{array}$ & $\begin{array}{r}70 \\
(+25 \%) \\
\end{array}$ & $\begin{array}{r}\mathbf{0} \\
(0 \%) \\
\end{array}$ & $\begin{array}{r}\mathbf{0} \\
(0 \%) \\
\end{array}$ & $\begin{array}{r}99 \\
(+32 \%) \\
\end{array}$ & $\begin{array}{r}\mathbf{9 6} \\
(+23 \%) \\
\end{array}$ & $\begin{array}{r}\mathbf{9 6} \\
(+35 \%)\end{array}$ \\
\hline & & total & $\begin{array}{r}444 \\
(+6 \%)\end{array}$ & $\begin{array}{r}470 \\
(+6 \%)\end{array}$ & $\begin{array}{r}470 \\
(+8 \%)\end{array}$ & $\begin{array}{r}480 \\
(+4 \%)\end{array}$ & $\begin{array}{r}409 \\
(0 \%)\end{array}$ & $\begin{array}{r}409 \\
(0 \%)\end{array}$ & $\begin{array}{r}464 \\
(+6 \%)\end{array}$ & $\begin{array}{r}\mathbf{4 5 0} \\
(+4 \%)\end{array}$ & $\begin{array}{r}450 \\
(+6 \%)\end{array}$ \\
\hline & \multirow{3}{*}{ mid } & inside & $\begin{array}{r}\mathbf{3 5 6} \\
(0 \%)\end{array}$ & $\begin{array}{r}364 \\
(0 \%) \\
\end{array}$ & $\begin{array}{r}364 \\
(0 \%)\end{array}$ & $\begin{array}{r}\mathbf{4 1 4} \\
(0 \%)\end{array}$ & $\begin{array}{r}\mathbf{4 1 4} \\
(0 \%)\end{array}$ & $\begin{array}{r}\mathbf{4 1 4} \\
(0 \%) \\
\end{array}$ & $\begin{array}{r}396 \\
(0 \%)\end{array}$ & $\begin{array}{r}\mathbf{3 7 9} \\
(0 \%)\end{array}$ & $\begin{array}{r}\mathbf{3 7 9} \\
(0 \%)\end{array}$ \\
\hline & & outside & $\begin{array}{r}182 \\
(+19 \%) \\
\end{array}$ & $\begin{array}{r}221 \\
(+13 \%) \\
\end{array}$ & $\begin{array}{r}221 \\
(+18 \%) \\
\end{array}$ & $\begin{array}{r}140 \\
(+17 \%) \\
\end{array}$ & $\begin{array}{r}4 \\
(0 \%) \\
\end{array}$ & $\begin{array}{r}\mathbf{4} \\
(0 \%) \\
\end{array}$ & $\begin{array}{r}172 \\
(+18 \%) \\
\end{array}$ & $\begin{array}{r}145 \\
(+12 \%) \\
\end{array}$ & $\begin{array}{r}145 \\
(+19 \%)\end{array}$ \\
\hline & & total & $\begin{array}{r}\mathbf{5 3 8} \\
(+5 \%)\end{array}$ & $\begin{array}{r}585 \\
(+4 \%)\end{array}$ & $\begin{array}{r}585 \\
(+6 \%)\end{array}$ & $\begin{array}{r}553 \\
(+4 \%)\end{array}$ & $\begin{array}{r}418 \\
(0 \%)\end{array}$ & $\begin{array}{r}418 \\
(0 \%)\end{array}$ & $\begin{array}{r}568 \\
(+4 \%)\end{array}$ & $\begin{array}{r}\mathbf{5 2 4} \\
(+3 \%)\end{array}$ & $\begin{array}{r}\mathbf{5 2 4} \\
(+4 \%)\end{array}$ \\
\hline & \multirow{3}{*}{ high } & inside & $\begin{array}{r}\mathbf{3 8 3} \\
(0 \%)\end{array}$ & $\begin{array}{r}394 \\
(0 \%) \\
\end{array}$ & $\begin{array}{r}394 \\
(0 \%) \\
\end{array}$ & $\begin{array}{r}417 \\
(+2 \%) \\
\end{array}$ & $\begin{array}{r}419 \\
(0 \%) \\
\end{array}$ & $\begin{array}{r}419 \\
(0 \%) \\
\end{array}$ & $\begin{array}{r}430 \\
(+2 \%) \\
\end{array}$ & $\begin{array}{r}\mathbf{4 0 4} \\
(0 \%) \\
\end{array}$ & $\begin{array}{r}\mathbf{4 0 4} \\
(0 \%) \\
\end{array}$ \\
\hline & & outside & $\begin{array}{r}\mathbf{2 6 8} \\
(+6 \%) \\
\end{array}$ & $\begin{array}{r}339 \\
(+5 \%) \\
\end{array}$ & $\begin{array}{r}339 \\
(+8 \%) \\
\end{array}$ & $\begin{array}{r}232 \\
(+3 \%) \\
\end{array}$ & $\begin{array}{r}\mathbf{2 4} \\
(0 \%) \\
\end{array}$ & $\begin{array}{r}\mathbf{2 4} \\
(0 \%) \\
\end{array}$ & $\begin{array}{r}258 \\
(+5 \%) \\
\end{array}$ & $\begin{array}{r}\mathbf{2 0 5} \\
(+5 \%) \\
\end{array}$ & $\begin{array}{r}205 \\
(+7 \%)\end{array}$ \\
\hline & & total & $\begin{array}{r}\mathbf{6 5 1} \\
(+3 \%)\end{array}$ & $\begin{array}{r}733 \\
(+3 \%)\end{array}$ & $\begin{array}{r}733 \\
(+4 \%)\end{array}$ & $\begin{array}{r}649 \\
(+2 \%)\end{array}$ & $\begin{array}{r}443 \\
(0 \%)\end{array}$ & $\begin{array}{r}443 \\
(0 \%)\end{array}$ & $\begin{array}{r}688 \\
(+3 \%)\end{array}$ & $\begin{array}{r}\mathbf{6 1 0} \\
(+2 \%)\end{array}$ & $\begin{array}{r}\mathbf{6 1 0} \\
(+2 \%)\end{array}$ \\
\hline \multirow{9}{*}{ high } & \multirow{3}{*}{ low } & inside & $\begin{array}{r}\mathbf{3 5 4} \\
(0 \%)\end{array}$ & $\begin{array}{r}359 \\
(0 \%) \\
\end{array}$ & $\begin{array}{r}361 \\
(0 \%) \\
\end{array}$ & $\begin{array}{r}\mathbf{4 0 7} \\
(+2 \%) \\
\end{array}$ & $\begin{array}{r}412 \\
(0 \%)\end{array}$ & $\begin{array}{r}413 \\
(0 \%) \\
\end{array}$ & $\begin{array}{r}387 \\
(0 \%) \\
\end{array}$ & $\begin{array}{r}\mathbf{3 7 0} \\
(0 \%)\end{array}$ & $\begin{array}{r}371 \\
(0 \%) \\
\end{array}$ \\
\hline & & outside & $\begin{array}{r}\mathbf{3 8 9} \\
(+4 \%) \\
\end{array}$ & $\begin{array}{r}487 \\
(+2 \%) \\
\end{array}$ & $\begin{array}{r}465 \\
(+3 \%) \\
\end{array}$ & $\begin{array}{r}323 \\
(0 \%) \\
\end{array}$ & $\begin{array}{r}\mathbf{5} \\
(0 \%) \\
\end{array}$ & $\begin{array}{r}\mathbf{5} \\
(0 \%) \\
\end{array}$ & $\begin{array}{r}378 \\
(+3 \%) \\
\end{array}$ & $\begin{array}{r}375 \\
(+2 \%) \\
\end{array}$ & $\begin{array}{r}359 \\
(+3 \%)\end{array}$ \\
\hline & & total & $\begin{array}{r}742 \\
(+2 \%)\end{array}$ & $\begin{array}{r}846 \\
(0 \%)\end{array}$ & $\begin{array}{r}826 \\
(+1 \%)\end{array}$ & $\begin{array}{r}730 \\
(+1 \%)\end{array}$ & $\begin{array}{r}417 \\
(0 \%)\end{array}$ & $\begin{array}{r}418 \\
(0 \%)\end{array}$ & $\begin{array}{r}764 \\
(+2 \%)\end{array}$ & $\begin{array}{r}745 \\
(0 \%)\end{array}$ & $\begin{array}{r}730 \\
(+1 \%)\end{array}$ \\
\hline & \multirow{3}{*}{ mid } & inside & $\begin{array}{r}\mathbf{3 5 6} \\
(0 \%)\end{array}$ & $\begin{array}{r}378 \\
(0 \%)\end{array}$ & $\begin{array}{r}379 \\
(0 \%)\end{array}$ & $\begin{array}{r}\mathbf{4 0 9} \\
(+1 \%)\end{array}$ & $\begin{array}{r}415 \\
(0 \%)\end{array}$ & $\begin{array}{r}416 \\
(0 \%)\end{array}$ & $\begin{array}{r}407 \\
(0 \%)\end{array}$ & $\begin{array}{r}\mathbf{3 8 9} \\
(0 \%)\end{array}$ & $\begin{array}{r}\mathbf{3 8 9} \\
(0 \%)\end{array}$ \\
\hline & & outside & $\begin{array}{r}\mathbf{5 1 8} \\
(+2 \%) \\
\end{array}$ & $\begin{array}{r}642 \\
(0 \%) \\
\end{array}$ & $\begin{array}{r}631 \\
(0 \%) \\
\end{array}$ & $\begin{array}{r}465 \\
(0 \%) \\
\end{array}$ & $\begin{array}{r}6 \\
(0 \%) \\
\end{array}$ & $\begin{array}{r}6 \\
(0 \%) \\
\end{array}$ & $\begin{array}{r}506 \\
(0 \%) \\
\end{array}$ & $\begin{array}{r}444 \\
(0 \%) \\
\end{array}$ & $\begin{array}{r}436 \\
(0 \%)\end{array}$ \\
\hline & & total & $\begin{array}{r}\mathbf{8 7 5} \\
(0 \%)\end{array}$ & $\begin{array}{l}1021 \\
(0 \%)\end{array}$ & $\begin{array}{l}1010 \\
(0 \%)\end{array}$ & $\begin{array}{r}874 \\
(0 \%)\end{array}$ & $\begin{array}{r}421 \\
(0 \%)\end{array}$ & $\begin{array}{r}422 \\
(0 \%)\end{array}$ & $\begin{array}{r}914 \\
(0 \%)\end{array}$ & $\begin{array}{r}833 \\
(0 \%)\end{array}$ & $\begin{array}{r}\mathbf{8 2 6} \\
(0 \%)\end{array}$ \\
\hline & \multirow{3}{*}{ high } & inside & $\begin{array}{r}\mathbf{3 5 2} \\
(0 \%)\end{array}$ & $\begin{array}{r}404 \\
(0 \%)\end{array}$ & $\begin{array}{r}408 \\
(0 \%)\end{array}$ & $\begin{array}{r}\mathbf{4 1 2} \\
(+1 \%)\end{array}$ & $\begin{array}{r}419 \\
(0 \%)\end{array}$ & $\begin{array}{r}418 \\
(0 \%)\end{array}$ & $\begin{array}{r}427 \\
(0 \%)\end{array}$ & $\begin{array}{r}\mathbf{4 1 0} \\
(0 \%)\end{array}$ & $\begin{array}{r}413 \\
(0 \%)\end{array}$ \\
\hline & & outside & $\begin{array}{r}\mathbf{6 6 8} \\
(0 \%) \\
\end{array}$ & $\begin{array}{r}825 \\
(0 \%) \\
\end{array}$ & $\begin{array}{r}816 \\
(0 \%) \\
\end{array}$ & $\begin{array}{r}651 \\
(0 \%) \\
\end{array}$ & $\begin{array}{r}\mathbf{2 4} \\
(0 \%) \\
\end{array}$ & $\begin{array}{r}25 \\
(0 \%) \\
\end{array}$ & $\begin{array}{r}663 \\
(0 \%) \\
\end{array}$ & $\begin{array}{r}522 \\
(0 \%) \\
\end{array}$ & $\begin{array}{r}\mathbf{5 1 7} \\
(0 \%)\end{array}$ \\
\hline & & total & $\begin{array}{r}\mathbf{1 0 2 0} \\
(0 \%)\end{array}$ & $\begin{array}{l}1229 \\
(0 \%)\end{array}$ & $\begin{array}{l}1224 \\
(0 \%)\end{array}$ & $\begin{array}{r}1063 \\
(+1 \%)\end{array}$ & $\begin{array}{r}444 \\
(0 \%)\end{array}$ & $\begin{array}{r}443 \\
(0 \%)\end{array}$ & $\begin{array}{l}1091 \\
(0 \%)\end{array}$ & $\begin{array}{r}933 \\
(0 \%)\end{array}$ & $\begin{array}{r}\mathbf{9 3 0} \\
(0 \%)\end{array}$ \\
\hline
\end{tabular}



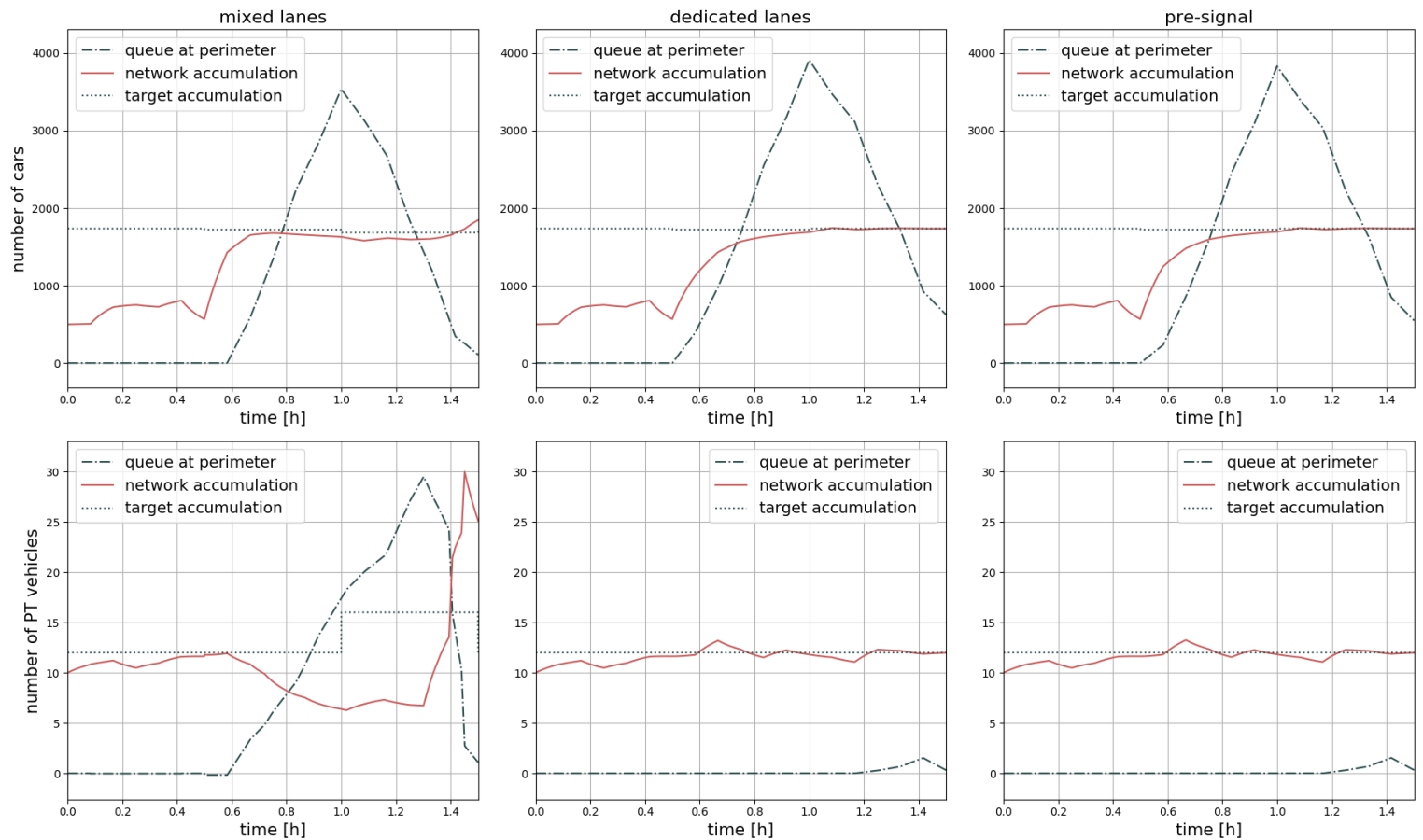

Figure B.10: Comparison of the three perimeter strategies in a realistic morning peak period considering noises: network states and control parameters for the case with high incoming car demand 20000veh/h and low incoming PT demand 100veh/h. 


\section{References}

Aboudolas, K., Geroliminis, N., 2013. Perimeter and boundary flow control in multi-reservoir heterogeneous networks. Transportation Research Part B: Methodological 55, 265-281.

Adler, M. W., van Ommeren, J. N., 2016. Does public transit reduce car travel externalities? quasi-natural experiments' evidence from transit strikes. Journal of Urban Economics 92, 106-119.

Ambühl, L., Loder, A., Becker, H., Menendez, M., Axhausen, K. W., 2018a. Evaluating london's congestion charge: An approach using the macroscopic fundamental diagram. In: 7th Transport Research Arena (TRA 2018).

Ambühl, L., Loder, A., Menendez, M., Axhausen, K. W., 2018b. A case study of zurichs two-layered perimeter control. In: 7th Transport Research Arena (TRA 2018).

Ampountolas, K., Zheng, N., Geroliminis, N., 2017. Macroscopic modelling and robust control of bi-modal multi-region urban road networks. Transportation Research Part B: Methodological.

Amsuess, S., Goebel, P., Graimann, B., Farina, D., 2015. A multi-class proportional myocontrol algorithm for upper limb prosthesis control: Validation in real-life scenarios on amputees. IEEE Transactions on Neural Systems and Rehabilitation Engineering 23 (5), 827-836.

Åström, K. J., Hägglund, T., 2006. Advanced PID control. ISA-The Instrumentation, Systems and Automation Society.

Chatman, D. G., Noland, R. B., 2011. Do public transport improvements increase agglomeration economies? a review of literature and an agenda for research. Transport Reviews 31 (6), 725-742.

Chiabaut, N., 2015. Evaluation of a multimodal urban arterial: The passenger macroscopic fundamental diagram. Transportation Research Part B: Methodological 81, 410-420.

Chiabaut, N., Küng, M., Menendez, M., Leclercq, L., 2018. Perimeter control as an alternative to dedicated bus lanes: A case study. Transportation Research Record 2672 (20), 110-120.

Daganzo, C. F., 2007. Urban gridlock: Macroscopic modeling and mitigation approaches. Transportation Research Part B: Methodological 41 (1), 49-62.

Daganzo, C. F., Geroliminis, N., 2008. An analytical approximation for the macroscopic fundamental diagram of urban traffic. Transportation Research Part B: Methodological 42 (9), 771-781.

Daganzo, C. F., Lehe, L. J., 2015. Distance-dependent congestion pricing for downtown zones. Transportation Research Part B: Methodological 75, 89-99.

Dakic, I., Ambühl, L., Schümperlin, O., Menendez, M., 2019a. On the modeling of passenger mobility for stochastic bi-modal urban corridors. Transportation Research Part C: Emerging Technologies (in press).

Dakic, I., Menendez, M., 2018. On the use of lagrangian observations from public transport and probe vehicles to estimate car space-mean speeds in bi-modal urban networks. Transportation Research Part C: Emerging Technologies 91, 317-334.

Dakic, I., Yang, K., Menendez, M., 2019b. Evaluating the effects of passenger occupancy dynamics on a bimodal perimeter control. In: TRB Annual Meeting Online, 19-05589, Washingtion, DC: Transportation Research Board.

Ge, Q., Ciuffo, B., Menendez, M., 2015. Combining screening and metamodel-based methods: An efficient sequential approach for the sensitivity analysis of model outputs. Reliability Engineering \& System Safety $134,334-344$. 
Ge, Q., Menendez, M., 2014. An efficient sensitivity analysis approach for computationally expensive microscopic traffic simulation models. International Journal of Transportation 2 (2), 49-64.

Ge, Q., Menendez, M., 2017. Extending morris method for qualitative global sensitivity analysis of models with dependent inputs. Reliability Engineering \& System Safety 162, 28-39.

Geroliminis, N., Daganzo, C. F., 2008. Existence of urban-scale macroscopic fundamental diagrams: Some experimental findings. Transportation Research Part B: Methodological 42 (9), 759-770.

Geroliminis, N., Haddad, J., Ramezani, M., 2013. Optimal perimeter control for two urban regions with macroscopic fundamental diagrams: A model predictive approach. IEEE Transactions on Intelligent Transportation Systems 14 (1), 348-359.

Geroliminis, N., Zheng, N., Ampountolas, K., 2014. A three-dimensional macroscopic fundamental diagram for mixed bi-modal urban networks. Transportation Research Part C: Emerging Technologies 42, 168-181.

Gonzales, E. J., Daganzo, C. F., 2012. Morning commute with competing modes and distributed demand: User equilibrium, system optimum, and pricing. Transportation Research Part B: Methodological 46 (10), $1519-1534$.

Guler, S. I., Cassidy, M. J., 2012. Strategies for sharing bottleneck capacity among buses and cars. Transportation Research Part B: Methodological 46 (10), 1334-1345.

Guler, S. I., Menendez, M., 2014a. Analytical formulation and empirical evaluation of pre-signals for bus priority. Transportation Research Part B: Methodological 64, 41-53.

Guler, S. I., Menendez, M., 2014b. Evaluation of presignals at oversaturated signalized intersections. Transportation Research Record: Journal of the Transportation Research Board (2418), 11-19.

Haddad, J., Zheng, Z., 2018. Adaptive perimeter control for multi-region accumulation-based models with state delays. Transportation Research Part B: Methodological.

He, H., Guler, S. I., Menendez, M., 2016. Adaptive control algorithm to provide bus priority with a pre-signal. Transportation Research Part C: Emerging Technologies 64, 28-44.

He, H., Menendez, M., Guler, S. I., 2018. Analytical evaluation of flexible-sharing strategies on multimodal arterials. Transportation Research Part A: Policy and Practice 114, 364-379.

Hensher, D. A., 2001. Measurement of the valuation of travel time savings. Journal of Transport Economics and Policy (JTEP) 35 (1), 71-98.

Keyvan-Ekbatani, M., Kouvelas, A., Papamichail, I., Papageorgiou, M., 2012. Exploiting the fundamental diagram of urban networks for feedback-based gating. Transportation Research Part B: Methodological 46 (10), 1393-1403.

Keyvan-Ekbatani, M., Yildirimoglu, M., Geroliminis, N., Papageorgiou, M., 2015. Multiple concentric gating traffic control in large-scale urban networks. IEEE Transactions on Intelligent Transportation Systems 16 (4), 2141-2154.

Kottenhoff, K., Freij, K. B., 2009. The role of public transport for feasibility and acceptability of congestion charging-the case of stockholm. Transportation Research Part A: Policy and Practice 43 (3), 297-305.

Li, L., Yang, K., Li, Z., Zhang, Z., 2013. The optimality condition of the multiple-cycle smoothed curve signal timing model. Transportation Research Part C: Emerging Technologies 27, 46-57.

Lindsey, R., Verhoef, E., 2001. Traffic congestion and congestion pricing. In: Handbook of transport systems and traffic control. Emerald Group Publishing Limited, pp. 77-105. 
Loder, A., Ambühl, L., Menendez, M., Axhausen, K. W., 2017. Empirics of multi-modal traffic networksusing the 3d macroscopic fundamental diagram. Transportation Research Part C: Emerging Technologies $82,88-101$.

Loder, A., Dakic, I., Bressan, L., Ambühl, L., Bliemer, M., Menendez, M., Axhausen, K. W., 2019. Capturing network properties with a functional form for the three-dimensional macroscopic fundamental diagram, transportation Research Part B: Methodological (under second review).

Mahmassani, H., Williams, J. C., Herman, R., 1987. Performance of urban traffic networks. In: Transportation and Traffic Theory (Proceedings of the Tenth International on Transportation and Traffic Theory Symposium, Cambridge, Massachusetts), NH Gartner, NHM Wilson, editors, Elsevier. pp. 1-18.

Mahmassani, H. S., Saberi, M., Zockaie, A., 2013. Urban network gridlock: Theory, characteristics, and dynamics. Transportation Research Part C: Emerging Technologies 36, 480-497.

Mariotte, G., Leclercq, L., 2019. Flow exchanges in multi-reservoir systems with spillbacks. Transportation Research Part B: Methodological 122, 327-349.

McMillan, G. K., Cameron, R. A., 2003. Models Unleashed: Virtual Plant and Model Predictive Control Applications. ISA-Instrumentation, Systems, and Automation Society.

Mohajerpoor, R., Saberi, M., Vu, H. L., Garoni, T. M., Ramezani, M., 2019. H $\infty$ robust perimeter flow control in urban networks with partial information feedback. Transportation Research Part B: Methodological.

Ortigosa, J., Zheng, N., Menendez, M., N. Geroliminis, 2017. Traffic performance and road space allocation in multimodal urban networks with an MFD representation. In: 96th Transportation Research Board Annual Meeting (TRB), Washington D.C.

Pandey, V., Boyles, S. D., 2018. Dynamic pricing for managed lanes with multiple entrances and exits. Transportation Research Part C: Emerging Technologies 96, 304-320.

Ramezani, M., Haddad, J., Geroliminis, N., 2015. Dynamics of heterogeneity in urban networks: aggregated traffic modeling and hierarchical control. Transportation Research Part B: Methodological 74, 1-19.

Santos, G., Bhakar, J., 2006. The impact of the London congestion charging scheme on the generalised cost of car commuters to the city of London from a value of travel time savings perspective. Transport Policy $13(1), 22-33$.

Shabani, H., Vahidi, B., Ebrahimpour, M., 2013. A robust pid controller based on imperialist competitive algorithm for load-frequency control of power systems. ISA transactions 52 (1), 88-95.

Smeed, R. J., 1961. The traffic problem in towns. In: Manchester Statistical Society. Vol. 8. pp. 1-59.

Smeed, R. J., 1968. Traffic studies and urban congestion. Journal of Transport Economics and policy, 33-70.

Wichiensin, M., Bell, M. G., Yang, H., 2007. Impact of congestion charging on the transit market: An inter-modal equilibrium model. Transportation Research Part A: Policy and Practice 41 (7), 703-713.

Yang, K., Guler, S. I., Menendez, M., 2016. Isolated intersection control for various levels of vehicle technology: Conventional, connected, and automated vehicles. Transportation Research Part C: Emerging Technologies 72, 109-129.

Yang, K., He, H., Menendez, M., 2018a. Bi-modal automated highway lanes: Control strategy and evaluation. In: 18th COTA International Conference of Transportation Professionals: Intelligence, Connectivity, and Mobility, CICTP 2018. American Society of Civil Engineers (ASCE), pp. 124-133. 
Yang, K., Menendez, M., Guler, S. I., 2019a. Implementing transit signal priority in a connected vehicle environment with and without bus stops. Transportmetrica B: Transport Dynamics 7 (1), 423-445.

Yang, K., Menendez, M., Zheng, N., 2019b. Heterogeneity aware urban traffic control in a connected vehicle environment: A joint framework for congestion pricing and perimeter control. Transportation Research Part C: Emerging Technologies 105, 439-455.

Yang, K., Zheng, N., Menendez, M., 2018b. Multi-scale perimeter control approach in a connected-vehicle environment. Transportation Research Part C: Emerging Technologies 94, 32-49.

Yu, W., Rosen, J., 2013. Neural pid control of robot manipulators with application to an upper limb exoskeleton. IEEE Transactions on cybernetics 43 (2), 673-684.

Zheng, N., Rérat, G., Geroliminis, N., 2016. Time-dependent area-based pricing for multimodal systems with heterogeneous users in an agent-based environment. Transportation Research Part C: Emerging Technologies 62, 133-148.

Zheng, N., Waraich, R. A., Axhausen, K. W., Geroliminis, N., 2012. A dynamic cordon pricing scheme combining the macroscopic fundamental diagram and an agent-based traffic model. Transportation Research Part A: Policy and Practice 46 (8), 1291-1303. 